\title{
Atmospheric abundances in post-AGB candidates of intermediate temperature ${ }^{\star, \star \star}$
}

\author{
A. Arellano Ferro ${ }^{1}$, Sunetra Giridhar ${ }^{2}$, and P. Mathias ${ }^{3}$ \\ 1 Instituto de Astronomía, Universidad Nacional Autónoma de México, Apdo. Postal 70-264, México D.F., \\ CP 04510, México \\ 2 Indian Institute of Astrophysics, Bangalore 560034, India \\ e-mail: giridhar@iiap.ernet.in \\ 3 Observatoire de la Côte d'Azur, Département Fresnel, UMR 6528, BP 4229, 06304 Nice Cedex 04, France \\ e-mail: mathias@obs-nice.fr
}

Received 23 August 2000 / Accepted 19 December 2000

\begin{abstract}
Detailed atmospheric abundances have been calculated for a sample of A-G supergiant stars with IR fluxes and/or high galactic latitudes. HD 172481 and HD 158616 show clear indications of being post-AGB stars that have experienced third dredge-up. HD 158616 is carbon-rich while the abundance pattern of HD 172481 and its large Li enhancement gives support to the hot bottom burning scenario that explains paucity of carbon-rich stars among AGB stars. HD 172324 is very likely a hot post-AGB star that shows a strong carbon deficiency. HD 725, HD 218753 and HD 331319 also appear to be evolved objects between the red giant and the AGB. HD 9167, HD 173638 with a few exceptions, reflect solar abundances and no signs of post red giant evolution. They are most likely young massive disk supergiants. Further analysis of proto-Planetary Nebula HDE 341617 reveals that He lines show signs of velocity stratification. The emission lines have weakened considerably since 1993. The envelope expands at $19 \mathrm{~km} \mathrm{~s}^{-1}$ relative to the star. Atmospheric abundances, evolutionary tracks and isochrones are used to estimate masses and ages of all stars in the sample.
\end{abstract}

Key words. stars: post-AGB - stars: chemically peculiar - stars: evolution

\section{Introduction}

The atmospheric chemical composition of post-asymptotic giant branch (post-AGB) stars and circumstellar environments is determined by nucleosynthesis and dredgeup events at the late AGB phases. At AGB, the star has a $\mathrm{C}-\mathrm{O}$ core surrounded by helium and hydrogen burning shells above which lies a deep convective envelope. The thermally pulsating phase (TP-AGB), though much shorter than early AGB phase (E-AGB), is responsible, through considerable mass-loss, for the ejection of large fraction of carbon and $s$-process elements into the ISM. At this phase, thermal pulses are caused by instabilities in the He-burning shell first discovered by Schwarzschild \& Härm (1965) and Weigert (1966). The excess energy generated by He shell flashes is transported by convection over the region that extends from the base of

\footnotetext{
Send offprint requests to: A. Arellano Ferro

e-mail: armando@astroscu.unam.mx

* Based on observations obtained at the Haute-Provence Observatory, France.

** Tables 11 and 12 are only available in electronic form at http://www. edpsciences.org
}

He-burning shell and the hydrogen-helium discontinuity. The He shell instabilities strongly influence the chemical composition of the convective envelope. The expansion and cooling of the intershell layers during a powerdown phase of the He shell flash causes the deepening of the convective envelope into regions containing the products of partial He-burning. The ${ }^{12} \mathrm{C},{ }^{19} \mathrm{~F}$ and the $s$-process elements are mixed into the outer envelope causing abundance variations at the surface of these stars. This process, known as the third dredge-up (TDU), is able to explain the formation of carbon stars (Busso et al. 1999), Wallerstein et al. (1997), Mowlavi (1999). At AGB the star is constantly losing mass, but a final phase of enhanced massloss by the superwind is believed to terminate the AGB phase producing a planetary nebula. Therefore, studying the chemical composition of the atmospheres and envelopes of evolved stars with IR fluxes, one expects to identify post-AGB stars and to provide important observational constraints for the theoretical work on nucleosynthesis, internal structure and mass-loss in evolved intermediate and low-mass stars. 
Post-AGB stars, as they evolve across the H-R diagram towards the white dwarf stage, form families of rather exotic objects like the $\mathrm{R}$ CrB stars, other subgroups of H-deficient and He-rich stars, planetary nebulae etc. In the $\mathrm{H}-\mathrm{R}$ diagram, they populate the region generally occupied by massive young supergiants evolving redwards from the main sequence, and having similar temperatures and luminosities. To differentiate the massive and young stars from the highly evolved low-mass post-AGB stars, detailed atmospheric abundance analysis is crucial.

Chemical analysis of high galactic latitude A-F supergiants have led to the discovery of many interesting postAGB stars such as HR 7671 (Luck et al. 1990), HR 4912 (Lambert et al. 1983), HR 4114 (Giridhar et al. 1997) or of selected IRAS sources such as IRAS $22223+4327$ and IRAS 04296+3429 (Decin et al. 1998). However, most of these high galactic latitude stars are field stars of unknown distances. It is therefore likely that a significant fraction of them could possibly turn out to be disk objects of nearly solar compositions. A search of post-AGB stars among high galactic latitude stars could be more rewarding if we put the additional constraint of IR detection. The wavelength dependence of IR fluxes and also the detection of submillimeter fluxes could give valuable information on the circumstellar matter surrounding the evolved star. The IRAS two colour diagrams such as those published by Olnon et al. (1984), van der Veen \& Habing (1988) etc., are extremely useful in separating stars with different kinds of envelopes.

In this study, we have undertaken the abundance analysis of a selected sample of stars likely to be post-AGB stars. From the published lists of high galactic latitude stars $\left(b>20^{\circ}\right)$ (e.g. Bidelman 1990 and others) we chose the ones with known infrared fluxes. Among them, the ones falling into the regions VIa and VIb of Fig. 5b of van der Veen \& Habing (1988) were preferred as they were more likely to be post-AGB stars. We have also included a few objects belonging to the regions IIIa and IIIb that are likely to be evolved stars with oxygen-rich envelopes.

The hot star HD 172324 was also included in spite of not being an IRAS source since it has high radial velocity $\left(-110 \mathrm{~km} \mathrm{~s}^{-1}\right)$ and very complex structures in hydrogen line profiles. It appeared to be a possible hot postAGB star similar to those investigated by Conlon et al. (1993a,b).

A search for post-AGB stars among supergiant-like stars of high galactic latitude is expected to be more efficient since the possibility of forming stars at truly large distances from the galactic plane is low.

This program is also aimed at providing calibrators for photometric empirical calibrations of atmospheric abundances (Arellano Ferro \& Mantegazza 1996), temperatures, and gravities in particular, since gravities are better determined from the ionization equilibrium.

This paper is organized in the following way: Sect. 2 describes the observations and data reduction; Sect. 3 discusses the methodology of abundance calculation; Sect. 4 gives an account of the sources of uncertainty in the derived abundances; in Sect. 5 the results are given and discussed for each star; in Sect. 6 these results are discussed in terms of the evolutionary status of each star while in Sect. 7 we summarize our results.

\section{Observations and data reduction}

The observational material for this work was obtained during July 6 - July 12, 1999 with the $1.93 \mathrm{~m}$ telescope of the Haute-Provence Observatory (OHP), which is equipped with the high resolution (42000) echelle spectrograph ELODIE. Details about the performance and characteristics of the instrument have been thoroughly described by Baranne et al. (1996). We have used one spectrum of HD 172324 taken on June 1995 with the Sandiford echelle spectrograph at $2.1 \mathrm{~m}$ telescope of McDonald Observatory. This instrument giving a resolution of 50000 has been described in McCarthy et al. (1993). One spectrum of HD 172481 was obtained with the $2.7 \mathrm{~m} \mathrm{2d}$ coude echelle spectrograph described in Tull et al. (1995). These spectra were reduced using spectroscopic data reduction tasks available in the IRAF package.

\subsection{The sample}

Table 1 contains the list of stars studied in this work, their spectral types, magnitudes, galactic positions and, when available, the IRAS infrared fluxes.

\subsection{Spectroscopic reductions}

All spectra were bias-subtracted and flat-field corrected using standard OHP procedures described by Baranne et al. (1996). The spectra were wavelength calibrated with Th-Ar hollow cathode lamp spectra taken after each stellar exposure. More than one exposure was taken and spectra were combined to attain $S / N$ of at least 50 . The ELODIE spectrograph gives a resolution of 42000 and the wavelength coverage goes from 3906 to $6811 \AA$ in 67 echelle orders with some overlaps in adjacent orders.

The equivalent widths were measured using the splot task of the IRAF package and their accuracy is generally better than $10 \%$ for spectra with $S / N$ ratio larger than 50 . We generally restricted ourselves to unblended weak features and avoided using lines stronger than $200 \mathrm{~m} \AA$.

\section{Abundance analysis}

We have used ATLAS9 (Kurucz 1993) model atmospheres as an input to the 1997 version of LTE line synthesis program MOOG first described in Sneden (1973). The procedure assumes plane-parallel atmospheres, hydrostatic equilibrium and LTE. The oscillator strength or $g f$ value is an important atomic datum that affects the abundance calculations. For elements $\mathrm{C}, \mathrm{N}$ and $\mathrm{O}$ we used $g f$ values from Wiese et al. (1996). For Fe I, the values were 
Table 1. Basic data and IRAS fluxes of sample stars

\begin{tabular}{|c|c|c|c|c|c|c|c|c|c|}
\hline Star & Sp.T. & $\begin{array}{c}V \\
(\mathrm{mag})\end{array}$ & $\begin{array}{c}l \\
\left(^{\circ}\right)\end{array}$ & $\begin{array}{c}b \\
\left(^{\circ}\right)\end{array}$ & IRAS & $\begin{array}{l}12 \mu \\
(\mathrm{Jy})\end{array}$ & $\begin{array}{l}25 \mu \\
(\mathrm{Jy})\end{array}$ & $\begin{array}{l}60 \mu \\
(\mathrm{Jy})\end{array}$ & $\begin{array}{c}100 \mu \\
(\mathrm{Jy})\end{array}$ \\
\hline HD 725 & F5Ib-II & 7.08 & 117.56 & -5.19 & $00091+5659$ & .36 & .25 & .40 & 14.43 \\
\hline HD 9167 & F1II & 8.19 & 127.73 & -0.97 & $01285+6115$ & .47 & .25 & .40 & 8.14 \\
\hline HD 158616 & F8 & 9.69 & 13.23 & +12.17 & $17279-1119$ & 3.52 & 2.90 & 1.60 & 1.98 \\
\hline HD 172324 & B9Ib & 8.16 & 66.18 & +18.58 & & & & & \\
\hline HD 172481 & $\mathrm{~F} 2 \mathrm{Ia} 0$ & 9.09 & 6.72 & -10.37 & $18384-2800$ & 5.41 & 5.22 & .59 & 1.85 \\
\hline HD 173638 & F2Ib-II & 5.73 & 23.38 & -3.56 & $18439-1010$ & 1.41 & .39 & .67 & 55.03 \\
\hline HD 218753 & A5III & 5.69 & 110.28 & -1.02 & & & & & \\
\hline HD 331319 & F3Ib & 9.50 & 67.16 & +2.73 & $19475+3119$ & .54 & 37.99 & 55.83 & 14.76 \\
\hline HDE 341617 & A5 & 9.40 & 50.67 & +19.79 & $18062+2410$ & 3.98 & 19.62 & 2.90 & 1.00 \\
\hline
\end{tabular}

taken in order of preference from; Table A1 of Lambert et al. (1996), Luck's compilation (1996, private communication), and Giridhar \& Arellano Ferro (1989). For Fe II lines we used the Table A2 of Lambert et al. (1996), Giridhar \& Arellano Ferro (1995) and Luck's compilation (1996, private communication).

For elements other than Fe, the large compilation by Luck (1996, private communication) was preferentially used and for some heavy $s$-process elements $g f$ values were taken from the work of Thévenin $(1989,1990)$.

\subsection{Determination of atmospheric parameters}

In addition to abundances, the line strengths are strongly affected by atmospheric parameters like the effective temperature $\left(T_{\text {eff }}\right)$, gravity $(\log g)$ and turbulent velocity $\left(\xi_{\mathrm{t}}\right)$. It is therefore necessary to determine these parameters before using line strengths for abundance determinations.

\subsubsection{Effective temperature}

Temperature calibrations exist that are valid for specific spectral type ranges. These methods are not only useful but also complement the spectroscopic efforts by providing initial values for the atmospheric parameters for calculating the atmospheric abundances. Here we briefly describe some of those calibrations that have been used for the stars of our sample. Later in Sect. 5, comparison with the finally adopted spectroscopic results is made in the discussion of each individual star.

Firstly, a rough estimate of $T_{\text {eff }}$ can be made from the given spectral type and the calibration of SchmidtKaler (1982). However, more accurate values can be obtained from precise photometric colours. For our sample, we have used uvby $\beta$ photometric data and our own unpublished calibrations for F-G stars (HD 725, HD 9167, HD 172481). In addition, the calibration of Napiwotzki et al. (1993) for hotter stars like HD 172324, the 13colour photometric system and the calibration of Bravo Alfaro et al. (1997), and the Geneva colour indices and the calibration of Cramer \& Maeder (1979) were used as appropriate. These empirical calibrations provide $T_{\text {eff }}$ with accuracies of $\pm 500 \mathrm{~K}$ or better and serve as excellent starting values that are further refined by spectroscopic approaches. Any drastic difference between the two approaches deserves attention.

Yet another independent approach to estimate $T_{\text {eff }}$ is from the Balmer lines profiles fitting (e.g. Arellano Ferro 1985; Venn 1995a). Theoretical Balmer profiles for grids of model atmospheres have been calculated by Kurucz (1993). For stars of intermediate temperature, this method does not lead to unique $T_{\text {eff }}$, but rather it defines loci of possible temperatures and gravities. On the other hand, similar loci can be found from species where two states of ionization are well represented. Again, the solution is not unique but rather a locus on the $T_{\text {eff }}-\log g$ plane is defined for each element. This approach will be illustrated in Fig. 3 for $\mathrm{H}_{\gamma}, \mathrm{H}_{\delta}, \mathrm{Mg}$ and $\mathrm{Si}$ for the star HD 218753. The above solution is of special importance for hot stars where no Fe I lines are present. For hotter stars, lines of Fe I are not only very weak but are also influenced by non-LTE effects. For A-F supergiants non-LTE effects could cause errors in the range of 0.2 to 0.3 dex in the iron abundance derived using Fe I lines (Boyarchuck et al. 1985). For example, in the well-known star Vega (A0V), the neglect of departure from LTE for Fe I lines leads to the underestimation of Fe abundance by 0.3 dex (Gigas 1986).

For stars cooler than $7500 \mathrm{~K}$ the lines of Fe I and Fe II with wide range in line strengths and lower excitation potential are adequate for estimating the effective temperature, microturbulence and gravity for any given star.

The finally adopted $T_{\text {eff }}$ for our stars is that for which abundance consistency is obtained from neutral and ionized lines of well represented species such as Fe, Ti and $\mathrm{Cr}$.

\subsubsection{Gravity}

A good discussion of atmospheric parameters determination for A type stars can be found in Venn (1995a), who points out that $\mathrm{H}_{\gamma}$ and $\mathrm{H}_{\delta}$, being very sensitive to temperature and gravity in A type stars, provide a locus of possible temperature-gravity pairs, and that ionisation equilibrium of $\mathrm{Mg} \mathrm{I}$ and $\mathrm{Mg}$ II gives another useful locus of temperature-gravity pair as the non-LTE effects are 
Table 2. Physical and dynamical parameters derived for program stars

\begin{tabular}{lccccccc}
\hline \multicolumn{1}{c}{ Star } & $\begin{array}{c}T_{\text {eff }} \\
(\mathrm{K})\end{array}$ & $\log g$ & $\begin{array}{c}\xi_{\mathrm{t}} \\
\left(\mathrm{km} \mathrm{s}^{-1}\right)\end{array}$ & $\begin{array}{c}V_{\mathrm{r}}(\mathrm{hel}) \\
\left(\mathrm{km} \mathrm{s}^{-1}\right)\end{array}$ & $\begin{array}{c}V(\mathrm{LSR}) \\
\left(\mathrm{km} \mathrm{s}^{-1}\right)\end{array}$ & $\begin{array}{c}\sigma_{V_{\mathrm{r}}} \\
\left(\mathrm{km} \mathrm{s}^{-1}\right)\end{array}$ & $\log (L / L \odot)$ \\
\hline HD 725 & 7000 & 1.0 & 4.65 & -56.9 & -48.8 & 0.8 & 4.2 \\
HD 9167 & 7250 & 0.5 & 4.20 & -45.7 & -40.2 & 1.1 & 4.2 \\
HD 158616 & 7300 & 1.5 & 4.6 & +68.8 & +78.6 & 2.1 & 4.5 \\
& & & & +63.7 & +78.5 & 1.5 & \\
HD 172324 & 11000 & 2.5 & 5.0 & -126.1 & -106.4 & 1.1 & 2.7 \\
& 11500 & 2.5 & 7.5 & -117.3 & -97.5 & 2.5 & \\
HD 172481 & 7250 & 1.5 & 4.60 & -73.1 & -76.9 & 1.8 & 4.1 .4 \\
& 7250 & 1.5 & 5.10 & -84.4 & -74.0 & 2.1 & 4.5 \\
HD 173638 & 7500 & 1.5 & 4.30 & +11.6 & +26.9 & 1.2 & 4.6 \\
HD 218753 & 8000 & 2.0 & 3.35 & +3.2 & +13.9 & 1.0 & 4.5 \\
HDE 341617 & 7750 & 1.0 & 5.35 & -2.5 & +15.8 & 1.5 & 4.6 \\
\hline
\end{tabular}

expected to be very small in magnesium lines. Since ionisation equilibrium of Si I and Si II give a temperaturegravity pair very similar to that given by $\mathrm{Mg}$ I and $\mathrm{Mg}$ II, the latter can also serve as yet another indicator of these parameters. Intersection of the above mentioned loci could lead to reliable temperature and gravity for each star, as demonstrated in Fig. 3.

The hydrogen lines were distorted in many of the program stars due to underlying emission, and therefore could not be used to derive temperature-gravity loci. We used excitation equilibrium of Fe I lines to get a preliminary estimate of $T_{\text {eff }}$. It was followed by ionisation equilibrium of $\mathrm{Mg} \mathrm{I} / \mathrm{Mg}$ II, Si I/Si II and Cr I/Cr II to arrive at a satisfactory estimate of $T_{\text {eff }}$ and $\log g$. For HD 725, HD 9167, HD 158616, HD 172481 and HD 173638 the excitation equilibrium of Fe I lines (requiring derived abundances to be independent of the lower excitation energy of the lines) gave very good estimates of the temperature which were further verified using lines of other species, as mentioned above. Similarly, for gravities, the values giving a good consistency for neutral and ionised $\mathrm{Mg}, \mathrm{Ti}, \mathrm{Cr}$ and Fe were adopted.

The star HD 172324 required an altogether different approach as described in Sect. 5.4.

\subsubsection{Microturbulence velocity}

All our program stars turned out to be hotter than $7000 \mathrm{~K}$ (see Table 2). For hotter stars, Fe I lines were difficult to measure. The Fe II lines on the other hand, had good range in equivalent widths. We therefore relied upon Fe II lines to derive microturbulence. The microturbulence was derived by requiring that weak, medium and strong lines give a consistent value of abundance.

The final atmospheric parameters derived for the program stars are given in Table 2, along with their radial velocities relative to the Sun and to the Local Standard of Rest (LSR). The $\log \left(L / L_{\odot}\right)$ values in Table 2 were estimated from the effective temperatures determined spectroscopically, and the calibration of Schmidt-Kaler (1982). For HD 172481 a red spectrum obtained at McDonald Observatory in May, 2000, allowed us to measure the three components of the OI feature near $7774 \AA$. The combined equivalent width $W(7774)=1.3 \AA$ and the calibration of Arellano Ferro et al. (1991) lead to $M_{\mathrm{v}}=$ -5.6 or $\log \left(L / L_{\odot}\right)=4.1$, which is in good agreement with the value 4.5 obtained from Schmidt-Kaler's calibration. For homogeneity we have adopted the latter value for our discussion about the evolutionary status of this object in Sect. 6 .

\section{Uncertainties in the elemental abundances}

The uncertainties in the derived abundances are caused by errors in the determination of the atmospheric parameters, in the equivalent width measurements, and also in the quality of oscillator strengths. For spectra with $S / N$ ratios larger than 50 the errors in the equivalent widths are between 5 and $8 \%$. The errors in $g f$ values vary from element to element. For Fe I lines, experimental values of good accuracies (better than 10\%) do exist, for other Fepeak elements the range in errors could be within 10 to $25 \%$. For heavier elements, particularly for $s$-process elements, the errors could be larger than $25 \%$. For the stars HD 725, HD 158616, HD 172481, HD 173638, HD 218753 and HD 331319 we could measure a very large number of unblended lines, and the estimated errors in $T_{\text {eff }}, \log g$ and $\xi_{\mathrm{t}}$ are $\pm 200 \mathrm{~K}, \pm 0.25$ and $\pm 0.2 \mathrm{~km} \mathrm{~s}^{-1}$, respectively. The sensitivity of the derived abundances to changes in the model atmospheric parameters are described in Table 4 of Gonzalez et al. (1997) for two RV Tau stars. We have used the same grid of atmospheric models and the same database for line oscillator strengths, hence the procedure will not be repeated here. For the Fe-peak elements we could measure a sufficiently large number of lines and the gf values used being of good quality, we expect these abundances to be accurate within 0.2 to 0.25 dex. For heavier elements, particularly the $s$-process elements, the 
Table 3. Elemental abundances for HD 725

\begin{tabular}{lcclrc}
\hline Species & $\log \epsilon_{\odot}$ & {$[\mathrm{X} / \mathrm{H}]$} & s.d. & $N$ & {$[\mathrm{X} / \mathrm{Fe}]$} \\
\hline C I & 8.55 & -0.38 & \pm 0.03 & 3 & -0.08 \\
Na I & 6.32 & +0.21 & \pm 0.08 & 3 & +0.51 \\
Mg I & 7.58 & -0.18 & \pm 0.25 & 4 & +0.12 \\
Si I & 7.55 & +0.05 & \pm 0.03 & 2 & +0.35 \\
Si II & 7.55 & +0.12 & & 1 & +0.42 \\
S I & 7.21 & -0.10 & \pm 0.17 & 3 & +0.20 \\
Ca I & 6.35 & -0.16 & \pm 0.10 & 12 & +0.14 \\
Sc II & 3.13 & -0.03 & \pm 0.23 & 7 & +0.27 \\
Ti II & 4.98 & -0.31 & \pm 0.08 & 6 & -0.02 \\
Cr I & 5.67 & -0.16 & \pm 0.17 & 7 & +0.14 \\
Cr II & 5.67 & -0.19 & \pm 0.16 & 11 & +0.11 \\
Mn I & 5.39 & -0.27 & \pm 0.12 & 6 & +0.03 \\
Fe I & 7.51 & -0.26 & \pm 0.14 & 51 & \\
Fe II & 7.51 & -0.33 & \pm 0.15 & 11 & \\
Ni I & 6.25 & +0.04 & \pm 0.14 & 3 & +0.33 \\
Y II & 2.23 & +0.12 & \pm 0.15 & 3 & +0.42 \\
Zr II & 2.60 & -0.07 & \pm 0.13 & 2 & +0.22 \\
Ba II & 2.13 & +0.15 & & 1 & +0.45 \\
Ce II & 1.58 & -0.17 & \pm 0.13 & 2 & +0.13 \\
\hline
\end{tabular}

Notes - The solar abundances are taken from Grevesse et al. (1996).

$-N$ is the number of lines included in the calculation.

uncertainty could be above 0.3 dex. Similarly for light elements like oxygen where few lines are available the uncertainty could be above 0.3 dex. For HD 172324 and HDE 341617, we will discuss the uncertainties in their respective sections.

\section{Results}

In what follows, we present derived elemental abundances for individual stars.

\subsection{HD 725}

This star is an IRAS source $(00091+5659)$. It was classified as F5Ib-II by Griffin \& Redman (1960) suggesting a temperature of $\sim 6900 \mathrm{~K}$ if we follow the calibration of Schmidt-Kaler (1982).

Using the uvby photometry of Perry (1969), Olsen (1983), Hauck \& Mermilliod (1998), reddening free colours and our own unpublished calibrations for F-G supergiant stars, we estimated $T_{\text {eff }}=6900 \mathrm{~K}$. Balmer line fitting was not performed because the profiles display complex structure and underlying emission is suspected.

The finally adopted parameters are $T_{\text {eff }}=7000 \mathrm{~K}$, $\log g=1.0$ and $\xi_{\mathrm{t}}=4.65 \mathrm{~km} \mathrm{~s}^{-1}$. We relied upon Fe II lines for calculating microturbulence velocity. A large number of Fe I lines were measured and used in estimat$\operatorname{ing} T_{\text {eff }}$. Also neutral and ionized lines of $\mathrm{Ti}$ and $\mathrm{Cr}$ were employed to derive a satisfactory pair of $T_{\text {eff }}$ and $\log g$ values. A competing solution could have been $T_{\text {eff }}=7200 \mathrm{~K}$, $\log g=1.5$ but the adopted parameters gave marginally better consistency in the abundances of neutral and ionized lines. HD 725 appears to be very marginally metalpoor (Table 3 ) and it has a moderately high radial velocity of $-57 \mathrm{~km} \mathrm{~s}^{-1}$. The elemental abundances relative to $\mathrm{Fe}$, i.e. $[\mathrm{X} / \mathrm{Fe}]=[\mathrm{X} / \mathrm{H}]-[\mathrm{Fe} / \mathrm{H}]^{1}$, given in the last column of Table 3 and subsequent tables, were calculated adopting the average value of $[\mathrm{Fe} / \mathrm{H}]$ from Fe I and Fe II lines. For HD 725 the $[\mathrm{X} / \mathrm{Fe}]$ values are similar to those in normal unevolved stars for many elements. $[\mathrm{Na} / \mathrm{Fe}]=+0.5 \mathrm{dex}$ indicates relative enrichment of $\mathrm{Na}$ which is a well-known feature of A-F supergiants (Takeda \& Takada-Hidai 1994; Venn 1995a,b). But Na I abundances are likely to be affected by non-LTE effect. Non-LTE analysis of Na I lines has been done by Gigas (1986), Takeda \& Takada-Hidai (1994) and others. The errors introduced by the neglect of non-LTE becomes more severe for higher temperatures. According to Takeda \& Takada-Hidai (1994), the $\triangle \log \epsilon$ in $\mathrm{Na}$ I lines at $5682,5688,6154$ and $6160 \AA$ is $-0.09,-0.10$, -0.07 and -0.07 dex respectively at temperature $7500 \mathrm{~K}$. At temperature $7000 \mathrm{~K}$ the correction is 0.01 dex smaller for all lines than the values mentioned above. According to Gigas (1986) the non-LTE correction could be +0.1 to +0.2 dex. The use of $[\mathrm{Na} / \mathrm{Ca}]$ instead of $[\mathrm{Na} / \mathrm{Fe}]$ is recommended by Lambert (1992) for LTE calculations. The $[\mathrm{Na} / \mathrm{Ca}]$ of +0.4 dex found from our analysis shows that $\mathrm{Na}$ enrichment appears to be real. The $\mathrm{Na}$ might have been synthesized in the H-burning region where the $\mathrm{NeNa}$ cycle might operate together with the CNO cycle. The suggestion that the proton capture on ${ }^{22} \mathrm{Ne}$ could lead to enhancement of ${ }^{23} \mathrm{Na}$ is followed up by Langer et al. (1993). These authors used a nuclear reaction network to examine the changes in abundances caused by proton capture at $T_{9}=0.040$. Mixing of this region just below the oxygen shell over a timescale of $30000 \mathrm{yr}$ would cause enhancement of ${ }^{14} \mathrm{~N},{ }^{23} \mathrm{Na}$ and ${ }^{27} \mathrm{Al}$ at the expense of ${ }^{16} \mathrm{O}$, ${ }^{22} \mathrm{Ne},{ }^{25} \mathrm{Mg}$ and ${ }^{26} \mathrm{Mg}$. These authors also suggested that the abundant ${ }^{20} \mathrm{Ne}$ could also be transformed into ${ }^{23} \mathrm{Na}$ on longer timescales, and pointed out that the depletion of ${ }^{25} \mathrm{Mg}$ and ${ }^{26} \mathrm{Mg}$ would not modify the $\mathrm{Mg}$ abundance. Globular cluster giants are known to display $\mathrm{Na}-\mathrm{O}$ anticorrelation as reported by Sneden et al. (1991) and Kraft et al. (1992). Since our spectrum does not go to wavelengths longer than $6800 \AA$, we could not measure N I abundance nor could we measure the $\mathrm{O}$ abundance. In metal-poor stars, relative enrichment of $\alpha$ elements is to be expected but the effect becomes evident for $[\mathrm{Fe} / \mathrm{H}]<$ -0.5 dex. At $[\mathrm{Fe} / \mathrm{H}]-0.2$ to -0.3 dex the spread in observed abundances is large and a large number of stars have $[\alpha / \mathrm{Fe}] \sim 0$. For HD 725, the $\alpha$ element Si shows small enhancement but the number of lines used are woefully small to make a definite claim.

Among Fe-peak elements, Ni, represented by $3 \mathrm{Ni}$ I lines, appears to be enriched, with $[\mathrm{Ni} / \mathrm{Fe}]$ of +0.3 dex. HD 218753 and HD 173638 of our sample show a positive $[\mathrm{Ni} / \mathrm{Fe}]$ but the value is not above abundance

\footnotetext{
${ }^{1}$ Using the spectroscopic notation $[\mathrm{X} / \mathrm{H}]=\log [(\mathrm{X} / \mathrm{H})$ $(\mathrm{X} / \mathrm{H}) \odot]$.
} 
errors. As such, for HR 725 the value is slightly above twice the standard error, nevertheless a more extensive analysis based on a larger number of lines is required to see if the enrichment is real. Luck \& Bond (1983, 1985) have reported $[\mathrm{Ni} / \mathrm{Fe}] \sim 0$ for metal-poor star. Wheeler et al. (1989) on the other hand report very large scatter in $[\mathrm{Fe} / \mathrm{H}]$ vs. $[\mathrm{Ni} / \mathrm{Fe}]$ relation. These authors suggest that non-zero $[\mathrm{Ni} / \mathrm{Fe}]$ can be observed at all metallicities.

Another interesting finding is $[\mathrm{Y} / \mathrm{Fe}]$ of $+0.4 \mathrm{dex}$. The three Y II lines used are quite well separated and have good estimates of oscillator strengths, but out of the three lines, one is a little strong. The same is true for the Ba II line used. Mild enrichment of $s$-process elements in the moderately metal-poor star HD 70379 has already been reported (Reddy 1996). Hence, our average $[\mathrm{s} / \mathrm{Fe}]$ ratio +0.3 does not come as a surprise. In addition, its radial velocity of $-57 \mathrm{~km} \mathrm{~s}^{-1}$ lends support to our view that it is a lowmass evolved object. These results are highly suggestive though not conclusive indicators of evolution beyond the red giant branch. However, making use of its proper motions and parallax from the Hipparcos catalogue we have calculated the galactocentric velocities $\Pi=-32.6 \mathrm{~km} \mathrm{~s}^{-1}$, $\Theta=+190.3 \mathrm{~km} \mathrm{~s}^{-1}$ and $Z=+5.4 \mathrm{~km} \mathrm{~s}^{-1}$ that indicate a space velocity of $193.2 \mathrm{~km} \mathrm{~s}^{-1}$ with a small pitch angle of $9.7^{\circ}$ relative to the circular orbit and on the galactic plane. This indicates that the star is at a lower galactic latitude than the Sun and placed in a mildly eccentric orbit.

\section{2. $H D 9167$}

This star has infrared flux and is an IRAS source $(01285+6115)$. It appears to be another moderately metalpoor star (Table 4). We did not find any remarkable abundance peculiarity for this object. With radial velocity of $-45 \mathrm{~km} \mathrm{~s}^{-1}$ and $[\mathrm{Fe} / \mathrm{H}]$ of $-0.3 \mathrm{dex}$, one could be optimistic of seeing positive $[\alpha / \mathrm{Fe}]$. Unfortunately, we could not measure good $\mathrm{Si}$ I and $\mathrm{Si}$ II lines and $\mathrm{Mg}, \mathrm{Ca}$ and Ti do not show any enrichment. But on the other hand, for stars with $[\mathrm{Fe} / \mathrm{H}]$ in the range of 0.0 to $-0.5 \mathrm{dex}$, the observed abundance ratios of $\alpha$-process elements have very large scatter, hence finding evolutionary changes is almost as probable as not finding them. Likewise HD 725, rather large radial velocity implies a space velocity of $208.2 \mathrm{~km} \mathrm{~s}^{-1}$ with a small pitch angle of $5.7^{\circ}$ relative to the circular orbit and on the galactic plane. The orbit is even less eccentric than that of HD 725 and it is at a lower galactic latitude than the Sun.

\section{3. $H D 158616$}

This star is an IRAS source (17279-1119) with significant infrared fluxes at shorter wavelengths.

The spectral type as listed in the SAO catalogue is F8, which suggests a temperature between 6100-6200 K (Schmidt-Kaler 1982), depending upon the luminosity class. No photometric data are available and hence no other temperature estimate was made. Two spectra were
Table 4. Elemental abundances for HD 9167

\begin{tabular}{lcclrl}
\hline Species & $\log \epsilon_{\odot}$ & {$[\mathrm{X} / \mathrm{H}]$} & s.d. & $N$ & {$[\mathrm{X} / \mathrm{Fe}]$} \\
\hline Mg I & 7.58 & -0.37 & \pm 0.15 & 2 & -0.04 \\
Ca I & 6.35 & -0.22 & \pm 0.09 & 6 & +0.12 \\
Sc II & 3.13 & -0.28 & \pm 0.09 & 3 & +0.05 \\
Ti II & 4.98 & -0.48 & \pm 0.17 & 10 & -0.15 \\
Cr I & 5.67 & -0.19 & & 1 & +0.15 \\
Cr II & 5.67 & -0.27 & \pm 0.21 & 9 & +0.07 \\
Mn I & 5.39 & -0.35 & & 1 & -0.02 \\
Fe I & 7.51 & -0.32 & \pm 0.16 & 29 & \\
Fe II & 7.51 & -0.35 & \pm 0.21 & 10 & \\
Y II & 2.23 & -0.23 & \pm 0.27 & 3 & +0.11 \\
Ba II & 2.13 & -0.22 & \pm 0.09 & 2 & +0.12 \\
\hline
\end{tabular}

Notes - same as Table 3.

obtained for this star at the OHP. The $S / N$ for these two spectra are 34 and 53 . Since the spectrum from July 7 is better, it was decided to use it to determine $T_{\text {eff }}, \log g$ and $\xi_{\mathrm{t}}$, complete the abundance analysis and then simply use the same parameters on the lower $S / N$ spectrum from July 6 to verify the abundance pattern. The results are given in Table 5 . The second entries for each species are for the lower $S / N$ spectrum. One can see that the results from both spectra are in a good agreement.

HD 158616 was also studied by Van Winckel (1995, 1997). While he had better data for C, N, O due to extended coverage in the long wavelength region, for other elements, our spectra contained more lines per element, and more elements are included. This star is Fe-poor by a factor of 4 or so and shows very clear indications of CNO processing and enrichment of $s$-process elements. We get $\mathrm{C} / \mathrm{O} \sim 1$ whereas Van Winckel (1995) got this ratio significantly larger than one (see Table 14). His carbon abundance is based on more lines in the $7100 \AA$ region. His choice of $T_{\text {eff }}$ is also hotter than our adopted value that can also account for the higher carbon abundance derived. Van Winckel (1995) found [N/Fe] of +0.2 dex. This clearly shows that the star has gone through $\mathrm{CNO}$ cycle and its products have been brought to the surface. The carbon enrichment could be caused by the helium-shell burning when oxygen is also manufactured. The enhancement of $\mathrm{Si}$ and $\mathrm{S}$ could be present in ISM from which the star is formed. With the $[\mathrm{Fe} / \mathrm{H}]$ of -0.6 dex, the star is moderately metal-poor and therefore it is possible that the parent ISM might have received ejecta from type II SNe. Although the effect of departure from LTE has been discussed for the light elements $\mathrm{C}, \mathrm{N}, \mathrm{O}$ and also for $\mathrm{Mg}$ by different investigators, we did not come across similar discussion on $\mathrm{Si}$ and $\mathrm{S}$. Hence, at this stage, we chose not to offer any detailed explanation. The most interesting feature of the derived abundances is definitely the enrichment of $s$-process elements Y, Ba, La and Ce. This star is undoubtedly a post-AGB star that has brought the products of helium burning as well as elements formed by $s$-processing to the surface. Our analysis covers the light 
Table 5. Elemental abundances for HD 158616

\begin{tabular}{lcrlrl}
\hline Species & $\log \epsilon \odot$ & {$[\mathrm{X} / \mathrm{H}]$} & \multicolumn{1}{l}{$s . d}$. & $N$ & {$[\mathrm{X} / \mathrm{Fe}]$} \\
\hline C I & 8.55 & -0.25 & \pm 0.20 & 4 & +0.33 \\
& & -0.23 & \pm 0.14 & 4 & +0.35 \\
O I & 8.87 & -0.54 & \pm 0.02 & 2 & +0.04 \\
& & -0.55 & & 1 & +0.03 \\
Na I & 6.32 & +0.05 & \pm 0.01 & 3 & +0.63 \\
& & -0.05 & & 1 & +0.49 \\
Mg I & 7.58 & -0.40 & \pm 0.11 & 3 & +0.18 \\
& & -0.56 & & 1 & -0.02 \\
Si I & 7.55 & +0.03 & \pm 0.17 & 3 & +0.61 \\
Si II & 7.55 & -0.06 & \pm 0.17 & 2 & +0.52 \\
& & +0.04 & \pm 0.47 & 2 & +0.58 \\
S I & 7.21 & +0.08 & \pm 0.12 & 6 & +0.66 \\
& & +0.10 & \pm 0.01 & 2 & +0.64 \\
Ca I & 6.35 & -0.36 & \pm 0.16 & 12 & +0.22 \\
& & -0.34 & \pm 0.31 & 9 & +0.20 \\
Sc II & 3.13 & +0.00 & \pm 0.27 & 8 & +0.58 \\
& & -0.09 & \pm 0.24 & 4 & +0.45 \\
Ti I & 4.98 & +0.08 & \pm 0.20 & 2 & +0.66 \\
& & +0.18 & & 1 & +0.72 \\
Ti II & 4.98 & -0.01 & \pm 0.19 & 13 & +0.57 \\
& & -0.13 & \pm 0.11 & 8 & +0.41 \\
Cr I & 5.67 & -0.55 & & 1 & +0.03 \\
Cr II & 5.67 & -0.39 & \pm 0.14 & 14 & +0.19 \\
& & -0.47 & \pm 0.22 & 7 & +0.07 \\
Mn I & 5.39 & -0.17 & & 1 & +0.41 \\
Fe I & 7.51 & -0.58 & \pm 0.15 & 39 & \\
& & -0.61 & \pm 0.18 & 20 & \\
Fe II & 7.51 & -0.57 & \pm 0.19 & 19 & \\
& & -0.51 & \pm 0.19 & 7 & \\
Ni I & 6.25 & -0.41 & \pm 0.08 & 4 & +0.17 \\
& & -0.39 & & 1 & +0.19 \\
Zn I & 4.60 & -0.48 & \pm 0.02 & 2 & +0.10 \\
& & -0.40 & & 1 & +0.18 \\
Y II & 2.23 & +0.37 & \pm 0.25 & 4 & +0.95 \\
& & +0.48 & \pm 0.03 & 2 & +1.02 \\
& 2.13 & +0.04 & \pm 0.22 & 3 & +0.62 \\
& & +0.10 & \pm 0.20 & 2 & +0.64 \\
& & -0.11 & & 1 & +0.47 \\
& & +0.13 & \pm 0.22 & 5 & +0.71 \\
& & & 1 & +0.75 \\
\hline & & & &
\end{tabular}

Notes - same as Table 3 .

- Second entries are results from a lower $S / N$ spectrum.

$s$-process element Y (one of the three elements referred as ls) and the heavy $s$-process elements Ba, La and Ce, representing heavy (hs) $s$-process elements. We have made an estimate of $[\mathrm{hs} / \mathrm{ls}]$. We have not corrected the ls-index for the lack of light elements $\mathrm{Sr}$ and $\mathrm{Zr}$ since for light elements the odd-even effect is not strong as pointed out by Van Winckel \& Reyniers 2000). We estimated [hs/ls] $=-0.3$ and $[\mathrm{ls} / \mathrm{Fe}]$ of +1.0 . When plotted on the $[\mathrm{hs} / \mathrm{ls}]$ vs. [ls $/ \mathrm{Fe}]$ plot of Busso et al. (1999), (their Fig. 7 giving theoretical predictions), we found $\tau_{0} \sim 0.22 \mathrm{mbarn}^{-1}$. Incidentally, the data point falls near the thick line which for solar metallicity would indicate $\mathrm{C} / \mathrm{O}=1$ but for $[\mathrm{Fe} / \mathrm{H}]$ of -0.6 ,

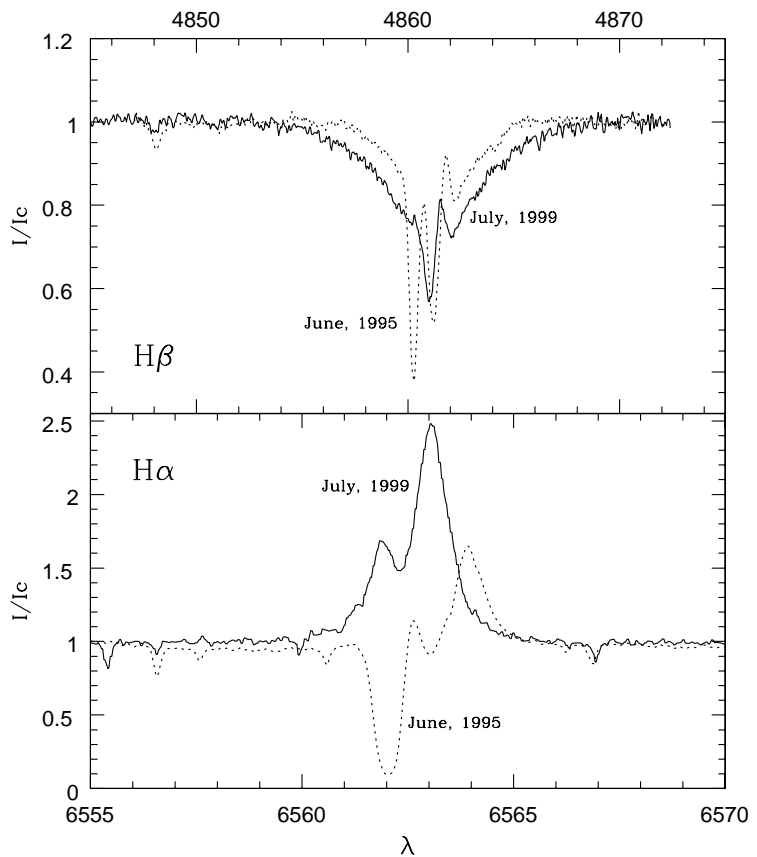

Fig. 1. $\mathrm{H}_{\alpha}$ and $\mathrm{H}_{\beta}$ variations exhibited by HD 172324 in two spectra taken 4 years apart: June 1995 at McDonald and July 1999 at OHP

it indicates a $\mathrm{C} / \mathrm{O} \sim 3$, whereas we get $\mathrm{C} / \mathrm{O} \sim 1$. This reduction of carbon abundance, while hs and ls indices point to larger $\mathrm{C} / \mathrm{O}$ added to small but significant enhancement of nitrogen, as reported by Van Winckel (1995), strongly favour the hot bottom burning scenario described and discussed in Sect. 5.5. More extensive coverage of $s$-process elements will enable a meaningful comparison with third dredge-up models developed by Straniero et al. (1995) and Busso et al. (1995).

Another star of similar temperature and showing a similar trend in abundances is HR 6144 (Luck et al. 1990), however, in this star, the $s$-process enhancement is not so significant and $\mathrm{C} / \mathrm{O}$ is less than 1.

\subsection{HD 172324}

This star has been classified as B9Ib by Morgan \& Roman (1950) which suggests a temperature of $10280 \mathrm{~K}$ (Schmidt-Kaler 1982). The Strömgren colours (Hauck \& Mermilliod 1998) however point towards higher temperature: $13100 \mathrm{~K}$ using the calibration of Napiwotzki et al. (1993). An independent temperature calibration of the Geneva photometric system (Cramer \& Maeder 1979) gives a temperature of $13215 \mathrm{~K}$.

The He I lines have been used to estimate $T_{\text {eff }}$ and luminosity class of the star. Didelon (1982) gives very useful plots of the dependence of many He I, Si II, Mg II, C II, O II and N II line strengths on the spectral type and luminosity class. The equivalent widths in our spectrum for the He I lines at 4120, 4143, 4387 and $4471 \AA$ very clearly suggest a spectral type of B9. A luminosity class Ib was suggested by the strengths of Si II line at $4128 \AA$, Mg II 
Table 6. Elemental abundances for HD 172324

\begin{tabular}{lcclrc}
\hline Species & $\log \epsilon_{\odot}$ & {$[\mathrm{X} / \mathrm{H}]$} & s.d. & $N$ & {$[\mathrm{X} / \mathrm{Fe}]$} \\
\hline He I & 10.99 & -0.08 & \pm 0.35 & 4 & +0.54 \\
He I* & 10.99 & -0.19 & \pm 0.35 & 12 & +0.44 \\
C II* $^{*}$ & 8.55 & -1.30 & & syn & -0.68 \\
O I & 8.87 & +0.41 & \pm 0.20 & 2 & +1.03 \\
O I* & 8.87 & +0.26 & \pm 0.19 & 4 & +0.89 \\
Ne I* & 8.09 & +0.05 & & 1 & +0.68 \\
Mg I* & 7.58 & -0.63 & & 1 & 0.00 \\
Mg II & 7.58 & -0.66 & \pm 0.17 & 2 & -0.04 \\
Mg II* & 7.58 & -0.65 & \pm 0.12 & 3 & -0.02 \\
Al II & 6.47 & -0.59 & & 1 & +0.03 \\
Al II* & 6.47 & -0.65 & & 1 & -0.02 \\
Si II & 7.55 & -0.16 & \pm 0.03 & 2 & +0.47 \\
Si II* & 7.55 & -0.29 & \pm 0.14 & 6 & +0.39 \\
Si III & 7.55 & +0.25 & & 1 & +0.88 \\
S II & 7.21 & -0.19 & & 1 & +0.43 \\
S II* & 7.21 & -0.47 & & 1 & +0.16 \\
Ti II & 4.98 & -0.03 & \pm 0.24 & 8 & +0.59 \\
Ti II* & 4.98 & -0.28 & \pm 0.33 & 3 & +0.35 \\
Cr II & 5.67 & -0.43 & \pm 0.19 & 11 & +0.19 \\
Cr II* & 5.67 & -0.48 & \pm 0.28 & 3 & +0.15 \\
Fe II & 7.51 & -0.62 & \pm 0.17 & 17 & \\
Fe II* & 7.51 & -0.63 & \pm 0.11 & 11 & \\
\hline
\end{tabular}

Notes - same as Table 3 .

$-T_{\text {eff }}=11000 \mathrm{~K}, \log g=2.5$ and $\xi_{\mathrm{t}}=7.00 \mathrm{~km} \mathrm{~s}^{-1}$ for $\mathrm{McD}$ spectrum.

$-T_{\text {eff }}=11500 \mathrm{~K}, \log g=2.5$ and $\xi_{\mathrm{t}}=5.40 \mathrm{~km} \mathrm{~s}^{-1}$ for OHP spectrum.

* Result from OHP spectrum.

line at $4481 \AA$ and C II feature at $4267 \AA$, this is in good agreement with the spectral type above.

An estimate of $T_{\text {eff }}$ has been made by requiring the He I lines to give solar abundance. The best estimate is $11500 \mathrm{~K}$. Given the class Ib, $\log g$ must not be very different from 2.0. In any case, at the high temperature end the Kurucz (1993) models do not reach very low gravities. Fortunately, Mg I and Mg II lines are present in the OHP spectrum and Si II and Si III lines can be measured on the McDonald spectrum. Our derived $T_{\text {eff }}$ and $\log g$ appear to be good estimates for the epoch of OHP spectrum. However there is indication that at the epoch at which the McDonald spectrum was taken, the gravity was somewhat lower.

This star appears to be deficient in Fe by a factor of $3-4$ and has a large radial velocity of $-110 \mathrm{~km} \mathrm{~s}^{-1}$ making it a likely halo or old disk object. Carbon abundance is very important in ascertaining the evolutionary status. We could observe only the blend at $4267 \AA$ while supposedly strong lines at 6578 and $6582 \AA$ were too weak to be measured. By computing the blend of two C II lines at $4267 \AA$ we find $[\mathrm{C} / \mathrm{H}]$ of $-1.3 \mathrm{dex}$, for which Takeda et al. (1996) found zero non-LTE correction. In the neighbourhood of $T_{\text {eff }} 10000 \mathrm{~K}$ the non-LTE abundance correction for carbon using C I lines is $\sim-0.4$ dex (Venn 1995b). Our iron abundance is based on Fe II lines that are not strongly affected by non-LTE effects. For O I line at $6156-$ $6158 \AA$ the non-LTE correction is $\sim-0.3$ dex (Takeda \& Takada-Hidai 1998).

For A-type supergiants of solar metallicity, Venn (1995a) found a mean enrichment of $\sim 0.6$ dex for $\mathrm{Na}$ and $\sim 0.3$ dex for S. Venn (1995b) reported CNO abundances for the same sample after applying non-LTE corrections to the derived abundances. The mean values found were: $[\mathrm{C} / \mathrm{H}]=-0.4 \operatorname{dex},[\mathrm{N} / \mathrm{H}]=+0.08 \operatorname{dex}$ and $[\mathrm{O} / \mathrm{H}]=$ -0.2 dex. Nevertheless, the hottest star of Venn's sample, HD 161695, is about $1500 \mathrm{~K}$ cooler than HD 172324.

McErlean et al. (1999) have plotted LTE and nonLTE line profiles for a range of temperatures $10000 \mathrm{~K}$ to $35000 \mathrm{~K}$. At $11000 \mathrm{~K}$ the non-LTE correction for C II lines at $4267 \AA$ is very small, in agreement with the prediction of Takeda et al. (1996). Hence our values $[\mathrm{C} / \mathrm{H}]=$ -1.3 dex and $[\mathrm{C} / \mathrm{Fe}]=-0.68$ dex are realistic estimates.

For an A-type star of solar metallicity, a mean value $[\mathrm{C} / \mathrm{H}]=-0.4$ was reported by Venn (1995b), whereas for $\mathrm{B}$ supergiants McErlean et al. (1999) report mean values of $[\mathrm{C} / \mathrm{H}]=-0.35$ dex and $[\mathrm{O} / \mathrm{H}]=-0.32$ dex. After applying non-LTE correction for oxygen we get $[\mathrm{O} / \mathrm{H}]$ of +0.1 dex and $[\mathrm{O} / \mathrm{Fe}]$ of +0.7 dex. Our derived carbon and oxygen abundances in Table 6 are much different from what is expected for a young B9 supergiant. It has been suggested by Boothroyd \& Sackmann (1999) that the carbon deficiency can also be caused by deep circulating mixing below the base of the convective envelope followed by cool bottom processing (CBP) of the $\mathrm{CNO}$ isotopes. They also showed that the CBP became more extensive at reduced metallicities or at low masses. The high radial velocity for HD 172324 (more than $100 \mathrm{~km} \mathrm{~s}^{-1}$ ) and significantly low $[\mathrm{Fe} / \mathrm{H}]$ makes it very likely that it has experienced CBP.

The only trace of an odd $Z$ element we could observe was a single line of $\mathrm{Al} \mathrm{I}$, providing $[\mathrm{Al} / \mathrm{Fe}] \sim 0$. One does not see $[\mathrm{Al} / \mathrm{Fe}] \sim+0.30$ dex reported by Edvardsson et al. (1993), but with single line being used in our study not much significance could be attached to our estimated $[\mathrm{Al} / \mathrm{Fe}]$.

Hot stars at high galactic latitude were studied by Conlon et al. (1993a) who found very large Fe and C deficiencies in them. But the stars studied by these authors are much hotter than HD 172324. Neverthless, the abundance pattern of HD 172324 is similar to those studied by Conlon et al. (1993a,b) and McCausland et al. (1992). This star being most likely an oxygen-rich post-AGB star deserves an extended analysis covering $\mathrm{CNO}$ and as significant spectral variations can be seen in four years (see Fig. 1), a monitoring of hydrogen line profiles over long time scales will be of interest.

\section{5. $H D 172481$}

This star shows light variations of very small amplitude, \pm 0.15 mag (Van Winckel 1995). The spectral energy distribution of this star has an unusual multi-peaked shape 
Table 7. Elemental abundances for HD 172481

\begin{tabular}{|c|c|c|c|c|c|}
\hline Species & $\log \epsilon_{\odot}$ & {$[\mathrm{X} / \mathrm{H}]$} & s.d. & $N$ & {$[\mathrm{X} / \mathrm{Fe}]$} \\
\hline Li I & 1.16 & $+2.54:$ & & 1 & $+3.16:$ \\
\hline C I & 8.55 & -0.62 & \pm 0.21 & 12 & -0.01 \\
\hline N I & 7.97 & -0.63 & \pm 0.10 & 3 & -0.02 \\
\hline O I & 8.87 & -0.58 & \pm 0.05 & 2 & +0.04 \\
\hline Mg I & 7.58 & -0.13 & \pm 0.21 & 2 & +0.48 \\
\hline Mg II & 7.58 & -0.06 & \pm 0.54 & 3 & +0.55 \\
\hline Si I & 7.55 & -0.05 & \pm 0.17 & 6 & +0.57 \\
\hline Si II & 7.55 & -0.10 & \pm 0.06 & 3 & +0.52 \\
\hline S I & 7.21 & -0.04 & \pm 0.16 & 7 & +0.58 \\
\hline K I & 5.12 & -0.28 & & 1 & +0.34 \\
\hline $\mathrm{Ca} \mathrm{I}$ & 6.35 & -0.28 & \pm 0.19 & 12 & +0.34 \\
\hline Sc II & 3.13 & -0.20 & \pm 0.23 & 6 & +0.41 \\
\hline $\mathrm{Ti} \mathrm{I}$ & 4.98 & -0.31 & & 1 & +0.31 \\
\hline Ti II & 4.98 & -0.28 & \pm 0.30 & 6 & +0.34 \\
\hline V II & 4.00 & -0.00 & \pm 0.14 & 2 & +0.62 \\
\hline Cr I & 5.67 & -0.34 & \pm 0.05 & 3 & +0.28 \\
\hline Cr II & 5.67 & -0.41 & \pm 0.16 & 10 & +0.21 \\
\hline Mn I & 5.39 & -0.51 & \pm 0.11 & 3 & +0.11 \\
\hline $\mathrm{Fe} I$ & 7.51 & -0.62 & \pm 0.15 & 43 & \\
\hline Fe II & 7.51 & -0.61 & \pm 0.14 & 13 & \\
\hline $\mathrm{Ni} \mathrm{I}$ & 6.25 & -0.31 & \pm 0.22 & 6 & +0.30 \\
\hline Zn I & 4.60 & -0.23 & \pm 0.08 & 2 & +0.39 \\
\hline Y II & 2.23 & +0.08 & \pm 0.26 & 4 & +0.69 \\
\hline Zr II & 2.60 & -0.14 & & 1 & +0.46 \\
\hline Ba II & 2.13 & +0.03 & \pm 0.20 & 2 & +0.65 \\
\hline La II & 1.21 & -0.55 & \pm 0.14 & 2 & +0.07 \\
\hline Ce II & 1.55 & -0.24 & \pm 0.19 & 7 & +0.38 \\
\hline Nd II & 1.50 & +0.03 & \pm 0.09 & 3 & +0.64 \\
\hline Eu II & 0.51 & +0.24 & & 1 & +0.86 \\
\hline
\end{tabular}

Note - same as Table 3.

(Bogart 1994). It was included in the study of Van Winckel (1995) who, by fitting the observed flux distribution to that of Kurucz (1993) model atmosphere, derived $T_{\text {eff }}=$ 7000 K. Van Winckel also found that the strength of emission components in hydrogen lines varied strongly. From the study of radial velocity variation spread over a decade, he reported that there is not enough evidence for the binarity of the star. However, his spectra show considerable line splitting attributed to the passage of a shock. Fortunately, we obtained the spectrum of HD 172481 when the atmosphere of the star was stable and therefore no line splitting was observed, as can be seen in the Fig. 2 .

Sadly, the $S / N$ ratio of our spectrum is not very high. We could measure very few lines of important elements like $\mathrm{C}$ and $\mathrm{O}$, hence we were not satisfied with the limited data to carry out abundance analysis. But it was known beyond doubt that the star is highly evolved since we could identify lines of C I and also saw Li I line at $6708 \AA$. However, the Li I feature was falling at the end of our CCD frame and was showing distinct doubling. One component coming nearest to $\mathrm{Li}$ I wavelength gave $\log \epsilon(\mathrm{Li})$ of 1.8. But $S / N$ being very poor at the end of the CCD frame, we decided to follow this object with more spectra. At our request, David Yong of McDonald Observatory

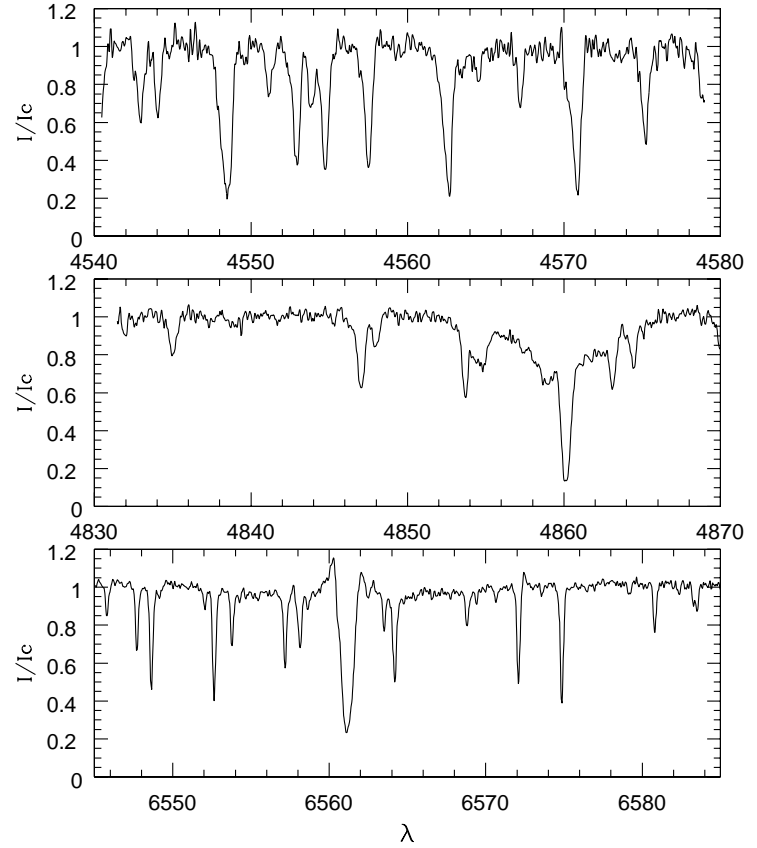

Fig. 2. Three spectral regions in the spectrum of HD 172481 clearly showing no line splitting. Hence we believe the star was in a stable phase

got a spectrum using the 2 d coudé echelle spectrograph at the $2.7 \mathrm{~m}$ telescope of McDonald Observatory on May 13, 2000. The spectrum has resolution of 30000 and wide spectral coverage from $3900 \AA$ to $10200 \AA$. In this spectrum, we found $\mathrm{Li}$ I feature to be single with some asymmetry in the blue wing but much deeper than in July 1999. In all HD 172481 spectra, the $\mathrm{H}_{\gamma}$ has a broad absorption, a narrow absorption and a possible red shifted emission component. The $\mathrm{H}_{\beta}$ also has a broad absorption and a narrow absorption at the centre of broad absorption. There is no indication of emission component. The $\mathrm{H}_{\alpha}$ has a complex profile with one shallow absorption, one deep absorption that has emission components in both the wings. The blue emission component is stronger than the red one.

The extensive spectral coverage of McDonald spectrum enabled us to measure a large number of unblended lines for several light and heavy elements. As one can see from the Table 7 , we could carry out a very comprehensive analysis of this object.

From Fe I and Fe II lines we derived $T_{\text {eff }}=7250 \mathrm{~K}$ and $\log g=1.5$ and was supported by Si I, Si II, Mg I and $\mathrm{Mg}$ II lines. We derived the $\mathrm{Li}$ abundance by spectrum synthesis. All the four components of Li I feature at the $6708 \AA$ region were included. We find $\log \epsilon(\mathrm{Li})$ of +3.7 , which is surprisingly high. We were puzzled by the change in appearance of $\mathrm{Li}$ I feature at 6707, so on August 11 one more spectrum was obtained with the same set-up and by the same observer. The spectrum again showed a suggestion of doubling at the line core though the components were not well separated. It is not clear if the line is splitting periodically or the emission at the core is causing 
it to appear double. The overall strength of Li I feature in the August spectrum has reduced. For May 2000 spectrum, our estimated Li abundance (derived by synthesis) is shown in Table 7 with a colon. In the light of the large variation exhibited by Li I feature in strength as well as in profile shape, this value should be regarded with caution. However, the presence of Li I feature in the spectrum cannot be refuted. Another Li I feature at $6103 \AA$ fell in between the echelle orders and therefore could not be used.

We could measure a large number of C I lines to estimate the carbon abundance. The three N I lines in the near infrared also enabled us to derive the nitrogen abundance. We get $[\mathrm{N} / \mathrm{Fe}] \sim 0$ for HD 172481 whereas for HD $158616[\mathrm{~N} / \mathrm{Fe}]=+0.3$ is reported by Van Winckel (1995). The estimated $\mathrm{C} / \mathrm{O}$ is +0.43 . The element $\mathrm{Li}$ is considered a fragile element that gets destroyed in the course of evolution. The primordial abundance of Li (based on population II objects) is considered near $\log \epsilon(\mathrm{Li})=2.2$. The excess abundance must therefore be caused by AGB evolution of HD 172481 or might owe its origin to binarity.

Our analysis covers light $s$-process elements (ls) Y and $\mathrm{Zr}$ and heavy $s$-process elements (hs) Ba, La, Ce and $\mathrm{Nd}$. We derive a mean $[\mathrm{ls} / \mathrm{Fe}]$ of 0.6 and mean $[\mathrm{hs} / \mathrm{Fe}]$ of 0.4 . That leads to $[\mathrm{hs} / \mathrm{ls}]$ of -0.2 . With this value of $[\mathrm{hs} / \mathrm{ls}]$ and $[\mathrm{Fe} / \mathrm{H}]$ of -0.6 this star is remarkably similar to IRAS 04296+3429 and IRAS 19500-1709 studied by Van Winckel \& Reyniers (2000), although [ls/Fe] and [hs/Fe] are much larger for these two stars.

The stars mentioned above and HD 158616 are very interesting post-AGB objects as they are, most likely, evolved from intermediate-mass stars (IMS). According to Travaglio et al. (1999), stars in the mass range $4-8 M_{\odot}$, activate ${ }^{22} \mathrm{Ne}(\alpha, n){ }^{25} \mathrm{Mg}$ reaction during their TP-AGB phase more effectively than the low-mass stars due to the high temperatures reached at the bottom of the convective pulse $\left(T_{\max } \leq 3.510^{8} \mathrm{~K}\right)$. IMS contribute more to the first $s$-peak represented by the elements $\mathrm{Sr}, \mathrm{Y}$ and Zr. Actually, the number of known post-AGB IMS is very small. For IMS, the formation of a ${ }^{13} \mathrm{C}$ pocket is less certain because of the reduced mass of the $\mathrm{H}-\mathrm{He}$ intershell by about one order of magnitude. It is shown by Straniero et al. (1997) and Gallino et al. (1998) that the ${ }^{13} \mathrm{C}$ neutron source active during the interpulse phase of low-mass TPAGB accounts for most of the production of the second $s$-peak elements Ba, La, Ce, Sm and Eu.

It is obvious from the relative enhancement of $\mathrm{Y}$ and Sr presented in Tables 5 and 7 that HD 158616 and HD 172481 belong to the relatively rare post-AGB IMS. We would therefore try to understand the observed abundances of HD 172481 in the framework of AGB models developed by Lattanzio and others for IMS.

Lattanzio (1997) in his AGB calculation has predicted that for stars more massive than $4 M_{\odot}$ the bottom of the convective envelope penetrates into the hotter regions of the envelope where proton captures already modified the original CNO composition. This is called "hot bottom burning" (HBB) and results in many
Table 8. Elemental abundances for HD 173638

\begin{tabular}{lcclrl}
\hline Species & $\log \epsilon_{\odot}$ & {$[\mathrm{X} / \mathrm{H}]$} & s.d. & $N$ & {$[\mathrm{X} / \mathrm{Fe}]$} \\
\hline C I & 8.55 & -0.16 & \pm 0.13 & 5 & -0.09 \\
Mg I & 7.58 & -0.05 & \pm 0.12 & 5 & +0.03 \\
Si I & 7.55 & +0.36 & \pm 0.12 & 2 & +0.44 \\
Si II & 7.55 & +0.41 & & 1 & +0.49 \\
Ca I & 6.35 & +0.04 & \pm 0.25 & 12 & +0.12 \\
Ca II & 6.35 & -0.03 & & 1 & +0.05 \\
Sc II & 3.13 & +0.16 & \pm 0.18 & 6 & +0.24 \\
Ti I & 4.98 & +0.01 & & 1 & +0.09 \\
Ti II & 4.98 & -0.1 & \pm 0.18 & 17 & -0.04 \\
Cr I & 5.67 & +0.08 & \pm 0.07 & 3 & +0.16 \\
Cr II & 5.67 & -0.02 & \pm 0.18 & 15 & +0.06 \\
Mn I & 5.39 & +0.00 & & 1 & -0.08 \\
Fe I & 7.51 & -0.07 & \pm 0.13 & 62 & \\
Fe II & 7.51 & -0.08 & \pm 0.14 & 19 & \\
Ni I & 6.25 & +0.06 & \pm 0.17 & 7 & +0.14 \\
Zn I & 4.60 & -0.06 & \pm 0.05 & 2 & +0.02 \\
Y II & 2.23 & +0.02 & \pm 0.22 & 5 & +0.10 \\
Zr II & 2.60 & -0.02 & & 1 & +0.06 \\
Ba II & 2.13 & +0.15 & \pm 0.04 & 2 & +0.23 \\
Ce II & 1.58 & +0.02 & & 1 & +0.10 \\
Nd II & 1.48 & +0.11 & & 1 & +0.19 \\
\hline
\end{tabular}

Note - same as Table 3.

important changes in chemical composition of the envelope. Lattanzio (1997) predicted the production of $\mathrm{Li}$ by the Cameron-Fowler mechanism operating at bottom of the envelope. Sackmann \& Boothroyd (1992) showed that $\log \epsilon(\mathrm{Li}) \sim 4.5$ could be produced in stars with bolometric magnitude between -6 and -7 when the temperature at the base of the convective envelope exceeds $5010^{6} \mathrm{~K}$. From the study of AGB stars in SMC and LMC, Smith et al. (1995) found them to have bolometric magnitudes in the range -6 to -7.2 and to show lithium values of $\log \epsilon(\mathrm{Li}) \sim 1.0-4.0$. However, these are cool luminous $\mathrm{S}$ stars.

Lattanzio (1997) also predicted the destruction of carbon via $\mathrm{CN}$ cycle that could prevent $\mathrm{C} / \mathrm{O}$ ratio from exceeding one. Theoretical models for AGB stars of mass 4, 5 and $6 M_{\odot}$ computed by Boothroyd et al. (1993) encountered HBB with a temperature at the base of the convective envelope reaching $8010^{6} \mathrm{~K}$. These models predict $\mathrm{C} / \mathrm{O}$ of 0.4 to 0.5 for $\sim 10^{3} \mathrm{yr}$ on the AGB.

The abundance pattern of HD 172481 bears some resemblance to those found for HR 7671 though the latter is more metal-poor. Interestingly, HR 7671 also shows the $\mathrm{Li} \mathrm{I}$ feature though $\mathrm{Li}$ is not as overabundant as in HD 172481. The estimated C/O for HR 7671 is 0.4 . The mean $[\alpha / \mathrm{Fe}]$ is lesser than what we find for HD 172481 but $[\mathrm{s} / \mathrm{Fe}]$ is comparable.

The existence of objects like HD 172481 and HR 7671 lend further support to the HBB scenario, put forward to explain the paucity of carbon-rich stars among AGB stars. The observed variation of Li I feature in HD 172481 makes it a very promising candidate for binarity search. 
While the present paper was under the refereeing process, the referee called our attention to the then unpublished work on HD 172481 by Reyniers \& Van Winckel (2001). Thus a comparison with their results is most appropriate. These authors have derived atmospheric parameters $\left(T_{\text {eff }}=7250 \mathrm{~K}\right.$ and $\left.\log g=1.5\right)$ that are in excellent agreement with those derived by us. A small difference in $\xi_{\mathrm{t}}$ of $0.6 \mathrm{~km} \mathrm{~s}^{-1}$ is seen. These authors also find a large lithium abundance similar to our finding. The $[\mathrm{Fe} / \mathrm{H}]$ and other Fe-peak elements have very good agreement. The $s$-process elements La, Nd and Eu however, show some disagreement. We have measured 3 lines of $\mathrm{Nd}$ II so we are surprised by the lower limits placed by these authors. For Eu, we measured a fairly clean line at $6645 \AA$ whereas Reyniers \& Van Winckel used spectrum synthesis. It should be noted that the oscillator strengths used by these authors are quite different from those employed by us, that can possibly explain the abundance differences for the $s$-process elements. The $g f$ values for the lines of these elements are known to have larger uncertainties compared to those of the Fe-peak elements. Another important finding by Reyniers \& Van Winckel is the detection of a red luminuous companion, found from the presence of $\mathrm{TiO}$ bands in the red and also from the observed spectral energy distribution. As mentioned above, the presence of the companion might explain the Li I feature variations observed by us.

\section{6. $H D 173638$}

This star (HR 7055) was observed in the 13-Colour photometric system and its temperature was calculated by various approaches by Bravo Alfaro et al. (1997). The photometric temperature estimates range between $7486 \mathrm{~K}$ and $8100 \mathrm{~K}$. The spectrum being of $S / N=95$, we could measure a large number of lines covering many important elements like C, $\alpha$-process elements and Fe-peak elements. From the ionisation equilibrium of Fe I/Fe II, Cr I/Cr II, Ti I/Ti II, Si I/Si II and even Ca I/Ca II the atmospheric parameters are well-determined and listed in Table 2.

The star appears to have near-solar abundances for most elements except $\mathrm{Si}$ which is overabundant by a factor of 3 (Table 8$)$. With its small radial velocity $\left(+11 \mathrm{~km} \mathrm{~s}^{-1}\right)$, it is most likely a young star belonging to the disk population. But it can serve as excellent calibrator of photometric indices in the $7500 \mathrm{~K}$ temperature range.

\subsection{HD 218753}

This star was classified as A5III by Cowley et al. (1969). These and the Sp.T. - Colour - $T_{\text {eff }}$ calibration of SchmidtKaler (1982) give a temperature of $8091 \mathrm{~K}$.

Photometric data in the Strömgren's system can also be used to estimate $T_{\text {eff }}$, through the $\beta-(b-y)$ relation of Crawford (1979) (his Table 1) and adopting $(b-y)=0.236$ and $\beta=2.773$ (Hauck \& Mermilliod 1998). This leads to $(b-y)_{0}=0.176$ and $E(b-y)=0.054$. While $(b-y)_{0}$ leads

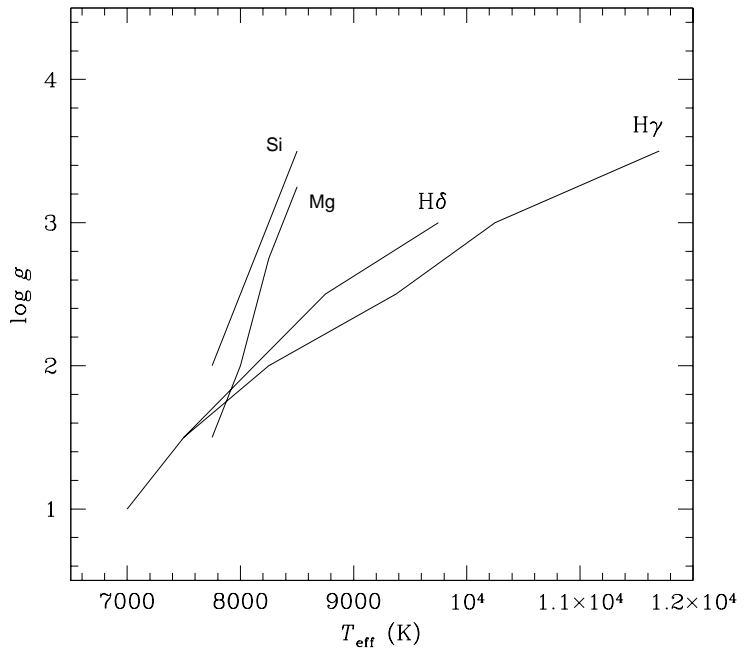

Fig. 3. $\mathrm{H}_{\gamma}, \mathrm{H}_{\delta}, \mathrm{Mg}$ and $\mathrm{Si}$ loci for $\mathrm{HD} 218753$

Table 9. Elemental abundances for HD 218753

\begin{tabular}{lrrlrl}
\hline Species & $\log \odot_{\odot}$ & {$[\mathrm{X} / \mathrm{H}]$} & s.d. & $N$ & {$[\mathrm{X} / \mathrm{Fe}]$} \\
\hline C I & 8.55 & -0.49 & \pm 0.18 & 5 & -0.30 \\
O I & 8.87 & -0.14 & & 1 & +0.05 \\
Na I & 6.32 & +0.32 & \pm 0.07 & 2 & +0.51 \\
Mg I & 7.58 & -0.27 & \pm 0.16 & 5 & -0.08 \\
Mg II & 7.58 & -0.29 & \pm 0.15 & 4 & -0.10 \\
Si I & 7.55 & +0.11 & \pm 0.15 & 3 & +0.20 \\
Si II & 7.55 & +0.00 & \pm 0.22 & 4 & +0.26 \\
S I & 7.21 & +0.17 & \pm 0.09 & 2 & +0.36 \\
Ca I & 6.35 & -0.08 & \pm 0.15 & 15 & +0.11 \\
Ca II & 6.35 & -0.09 & & 1 & +0.10 \\
Sc II & 3.13 & +0.00 & \pm 0.27 & 8 & -0.19 \\
Ti I & 4.98 & -0.27 & & 1 & -0.08 \\
Ti II & 4.98 & -0.25 & \pm 0.17 & 19 & -0.06 \\
Cr I & 5.67 & -0.01 & \pm 0.38 & 3 & +0.18 \\
Cr II & 5.67 & -0.16 & \pm 0.13 & 14 & +0.03 \\
Mn I & 5.39 & -0.23 & \pm 0.06 & 3 & -0.04 \\
Fe I & 7.51 & -0.20 & \pm 0.16 & 82 & \\
Fe II & 7.51 & -0.18 & \pm 0.13 & 30 & \\
Ni I & 6.25 & -0.06 & \pm 0.15 & 9 & +0.13 \\
Zn I & 4.60 & +0.18 & & 1 & +0.37 \\
Y II & 2.23 & -0.24 & \pm 0.21 & 7 & -0.05 \\
Zr II & 2.60 & +0.18 & & 1 & +0.37 \\
Ba II & 2.13 & -0.08 & \pm 0.16 & 2 & +0.11 \\
\hline
\end{tabular}

Notes - same as Table 3 .

to $(B-V)_{0}=0.20-0.25$ (Crawford 1970), this implies $T_{\text {eff }}=7800 \mathrm{~K}$.

Also, if the above colour excess and photometry are used in combination of Napiwotzki et al. (1993) calibration we find $T_{\text {eff }}=7200 \mathrm{~K}$. However, it must be emphasized that the calibration has been computed using only stars of luminosity classes V and IV, while HD 218753 could be of luminosity class III or II.

Balmer line fitting can also be used to estimate $T_{\text {eff }}$. Theoretical profiles have been extracted from Kurucz's (1993) models for $\mathrm{H}_{\alpha}, \mathrm{H}_{\beta}, \mathrm{H}_{\gamma}$ and $\mathrm{H}_{\delta}$. The observed 
Table 10. Elemental abundances for HD 331319

\begin{tabular}{lcclrl}
\hline Species & $\log \epsilon_{\odot}$ & {$[\mathrm{X} / \mathrm{H}]$} & s.d. & $N$ & {$[\mathrm{X} / \mathrm{Fe}]$} \\
\hline C I & 8.55 & -0.33 & \pm 0.30 & 5 & -0.09 \\
O I & 8.87 & +0.06 & \pm 0.02 & 2 & +0.30 \\
Na I & 6.32 & -0.03 & & 1 & +0.21 \\
Mg I & 7.58 & -0.32 & \pm 0.21 & 3 & -0.08 \\
Mg II & 7.58 & -0.18 & \pm 0.12 & 3 & +0.06 \\
Al I & 6.47 & +0.07 & & 1 & +0.31 \\
Si II & 7.55 & -0.18 & \pm 0.20 & 2 & +0.06 \\
S I & 7.21 & +0.29 & \pm 0.18 & 5 & +0.53 \\
Ca I & 6.35 & -0.29 & \pm 0.18 & 10 & -0.05 \\
Sc II & 3.13 & -0.19 & \pm 0.22 & 5 & +0.05 \\
Ti II & 4.98 & -0.33 & \pm 0.22 & 22 & -0.09 \\
V II & 4.01 & +0.19 & \pm 0.16 & 3 & +0.43 \\
Cr I & 5.67 & -0.01 & \pm 0.29 & 3 & +0.23 \\
Cr II & 5.67 & +0.02 & \pm 0.14 & 18 & +0.22 \\
Mn I & 5.39 & -0.17 & & 1 & +0.07 \\
Fe I & 7.51 & -0.27 & \pm 0.16 & 48 & \\
Fe II & 7.51 & -0.20 & \pm 0.15 & 21 & \\
Ni I & 6.25 & +0.00 & \pm 0.22 & 4 & -0.24 \\
Ni II & 6.25 & -0.13 & & 1 & +0.11 \\
Sr II & 2.90 & +0.01 & & 1 & +0.25 \\
Y II & 2.23 & -0.58 & \pm 0.09 & 3 & -0.34 \\
Ba II & 2.13 & -0.51 & \pm 0.16 & 3 & -0.27 \\
\hline
\end{tabular}

Notes - same as Table 3.

profiles were fitted with theoretical profiles of given $T_{\text {eff }}$ and $\log g$. The solution is not unique but in fact the best fits define a locus on the $T_{\text {eff }}-\log g$ plane for each Balmer line. As underlying core emission may be present in some stars, we chose to fit the wings $\mathrm{H}_{\gamma}$ and $\mathrm{H}_{\delta}$. Following the above fitting process we found the loci for $\mathrm{H}_{\gamma}$ and $\mathrm{H}_{\delta}$ profiles shown in Fig. 3 .

In hot stars, magnesium and silicon lines can be used as $T_{\text {eff }}$ indicators (since Fe I lines are strongly affected by non-LTE effects). Given a pair $\left(T_{\text {eff }}, \log g\right)$ one searches for abundance consistency between neutral and ionized lines of $\mathrm{Mg}$ and $\mathrm{Si}$. Again, the solution is not unique but rather a locus on the $T_{\text {eff }}-\log g$ plane is defined for each element, as illustrated in Fig. 3. The intersections of the $\mathrm{Mg}$ and $\mathrm{Si}$ loci with the $\mathrm{H}_{\gamma}$ and $\mathrm{H}_{\delta}$ loci point to the proper temperature and gravity. In this fashion we estimated $T_{\text {eff }}$ between 7600 and $7900 \mathrm{~K}$ and $\log g$ between 1.75 and 2.0.

The turbulent velocity, $\xi_{\mathrm{t}}$, was estimated from Fe II lines by requiring abundance to be independent of line strength. We found $\xi_{\mathrm{t}}=3.3 \mathrm{~km} \mathrm{~s}^{-1}$.

For HD 218753 we finally adopted $T_{\text {eff }}=8000 \mathrm{~K}$, $\log g=2.0$ dex and $\xi_{\mathrm{t}}=3.3 \mathrm{~km} \mathrm{~s}^{-1}$. With these parameters the abundances for the rest of the detected species were calculated and the results are given in Table 9.

For this star our spectrum has $S / N=67$ and we could measure a large number of clean weak lines for most important elements. HD 218753 shows a significant carbon deficiency most likely caused by CNO processing. There is also a significant enrichment of sodium that has been found in many A-F supergiants as discussed before for
HD 725 (Takeda \& Takada-Hidai 1994). Among $\alpha$-capture elements, only S shows enrichment above detection limit. These are indications of the star having experienced the first dredge-up (e.g. $[\mathrm{C} / \mathrm{Fe}]=-0.3$ dex). However its position in the $\mathrm{H}-\mathrm{R}$ diagram of Fig. 5 is consistent with a $M \sim 1.5-2 M_{\odot}$ and an age of about $7.910^{8} \mathrm{yr}$.

\section{8. $H D 331319$}

This star is an IRAS source $(19475+3119)$. The IR fluxes are quite large (Table 1 ), the heliocentric radial velocity is very small $\left(-2.5 \mathrm{~km} \mathrm{~s}^{-1}\right)$. With a spectrum of $S / N=98$ we could measure a large number of lines and hence derive the atmospheric parameters as well as abundances with good accuracy (Table 10). The star is Fe-poor by about 1.8 times, but shows significant enrichment of sulphur. The derived abundances are based on 5 good lines hence the derived sulphur abundance cannot be ascribed to measurement errors. Similarly, vanadium also shows some enrichment. Sulphur enrichment appears to be a common feature of our sample stars. The derived abundance of carbon for HD 331319 clearly indicates the effect of $\mathrm{CN}$ processing. The star is most likely a young massive disk supergiant or bright giant ascending the red giant branch. The IR fluxes are probably caused by material ejected at this evolutionary stage.

\subsection{The proto-Planetary Nebula HDE 341617}

It is an interesting object that has been showing relatively fast changes in temperature and brightness. It has faded from $m_{\mathrm{v}}=8.8$ reported in BD catalogue to $m_{\mathrm{v}}=11.4$ estimated by Stephenson (1986). Extensive photometry was taken up by Arkhipova et al. (1999), who found rapid light variations with an amplitude of up to 0.3 mag during 1996-1997. These authors also obtained a spectrum and describe the absorption and emission lines present. The light variations of HDE 341617 and spectral appearance confirmed the suggestion of Volk \& Kwok (1989), based on IRAS color indices, that it is a candidate postAGB star. From spectral type A5 given in HDE, it had changed to class Be (1986) as reported by Downes \& Keyes (1988). A very extensive spectral investigation by Parthasarathy et al. (2000) has shown the star to have $T_{\text {eff }}=22000 \mathrm{~K}$ with large deficiency of carbon. These authors derived $T_{\mathrm{e}}$ of $10000 \mathrm{~K}$ and $N_{\mathrm{e}}$ of $2.510^{4} \mathrm{~cm}^{-3}$ for the nebula from the study of emission lines. The spectra used by Parthasarathy et al. were taken in 1993. As this object presents rapid variations, we felt we could examine the spectrum at our epoch (1999) and study the changes. A comparison of the emission features common to Parthasarathy et al. (2000) 1993 spectrum and ours from 1999 is presented in Fig. 4. Figure 4a shows that in 1999 the emission lines were weaker while Fig. 4b shows that the differences in equivalent widths are not a function of wavelength. Unfortunately, we did not observe any flux standard and so were unable to measure the fluxes in the 

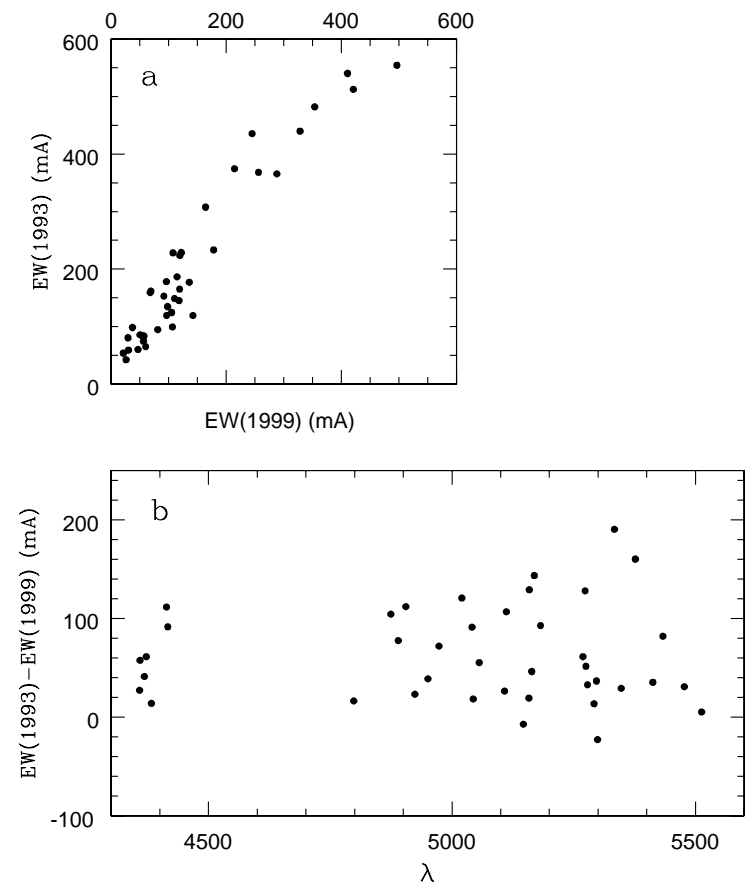

Fig. 4. A comparison of equivalent widths of the emission spectrum of HDE 341617 as observed in 1993 and in 1999. a) The weakening of the emission spectrum is evident despite the fact that the 1999 spectra have not been flux calibrated. b) The equivalent widths are not a function of wavelength. However, the large scatter is probably due to the lack of calibration. See text for discussion

emission lines. The shape of the pseudo continuum used for each echelle order may also be affected by instrumental sensitivity function. The large scatter is due to this fact. Weakening of lines (if it is not caused by resolution difference) might indicate further increase in temperature of the central star. Systematic monitoring of this fast evolving object could be very rewarding.

According to Arkhipova et al. (1999) HDE 341617 has a mass of $0.7 M_{\odot}$ with an envelope of $\sim 10^{-3} M_{\odot}$ undergoing rapid evolution towards the $\mathrm{PN}$ phase which, according to the predictions from Blöcker's (1995) models, should be reached within 100 years. This and the rapid photometric variations reported by Arkhipova et al. (1999), most likely caused by variations in the stellar wind, make the star a very interesting target for continuous monitoring.

We have two relatively low $S / N$ spectra that were used for identification and measurement of absortion and emission lines. We present in Tables 11 and 12 the line strengths and velocities for absorption and emission lines for this object.

Several absortion lines of He I, O II, Si III and Fe II are detected. The radial velocity, for each individual line unambiguously identified is given in Table 11. For He I two groups of lines are identified at average velocities of $52.6 \pm$ $8.3 \mathrm{~km} \mathrm{~s}^{-1}$ (4 lines) and $75.0 \pm 3.7 \mathrm{~km} \mathrm{~s}^{-1}$ (4 lines). This suggests some stratification in the atmosphere. The rest of the species however average $73.3 \pm 2.9 \mathrm{~km} \mathrm{~s}^{-1}$ (15 lines). The low dispersion in the radial velocity suggests that all
Table 13. Elemental abundances for the central star of the PPN HDE 341617

\begin{tabular}{lcccr}
\hline Species & $\log \epsilon_{\odot}$ & {$[\mathrm{X} / \mathrm{H}]$} & s.d. & $N$ \\
\hline C II & 8.55 & -1.43 & & 1 \\
N II & 7.97 & -0.50 & \pm 0.30 & 3 \\
O II & 8.87 & -0.51 & \pm 0.21 & 14 \\
Mg II & 7.58 & -1.13 & & 1 \\
Si III & 7.55 & -0.63 & \pm 0.06 & 2 \\
\hline
\end{tabular}

Notes - same as Table 3.

these lines are formed in the same region of the stellar atmosphere and no stratification is evident.

The emission spectrum, formed in the outer envelope and/or in the nebulosity, consists of lines of Fe II, [Fe II], Fe III, [S II], O I, [N II] and Si II. The radial velocities of emission lines are all very consistent and average $54.3 \pm 3.4 \mathrm{~km} \mathrm{~s}^{-1}$. The difference of radial velocities between the absorption and emission lines indicates that the nebulosity expands at about $19 \mathrm{~km} \mathrm{~s}^{-1}$. The Balmer lines show emission on top of the stellar absorption. The emission is shifted relative to the absorption and is consistent with the radial velocities of other emission features, showing that it is also produced in the same region.

Though the number of absorption lines were relatively small, we have done an abundance analysis for a few elements. The O II lines were used for fixing the microturbulence velocity. Since the line data was not adequate to do a detailed excitation equilibrium, we chose to use the temperature and gravity estimated by Parthasarathy et al. (2000) as starting value and tried models both hotter and cooler than their estimate. We got more consistent values for $T_{\text {eff }}=23000 \mathrm{~K}, \log g=3.0$ dex and $\xi_{\mathrm{t}}=15.0 \mathrm{~km} \mathrm{~s}^{-1}$ and hence these parameters were adopted although available lines were not particularly sensitive to the temperature. Temperature errors of $\pm 500 \mathrm{~K}$ or more are possible. With these parameters the atmospheric chemical abundances for the central star are those reported in Table 13.

\section{Discussion}

In order to estimate the mass and the age of each of the program stars we have plotted them on the $\mathrm{H}-\mathrm{R}$ diagram as can be seen in Fig. 5. The effective temperatures are those derived from the chemical analysis, and the luminosities were obtained from the above temperatures and the calibration of Schmidt-Kaler (1982); these quantities are listed in Table 2. Despite parallaxes do exist in the Hipparcos catalogue for some of the stars in our sample, we have preferred the above approach for the luminosity calculation for the following reasons. While a very respected version of the Period-Luminosity relation for cepheids has been calculated using Hipparcos parallaxes (Feast \& Catchpole 1997) using the most well-known 26 cepheids as calibrators, individual parallaxes seem to be of little use. We have taken 21 cepheids such that the 

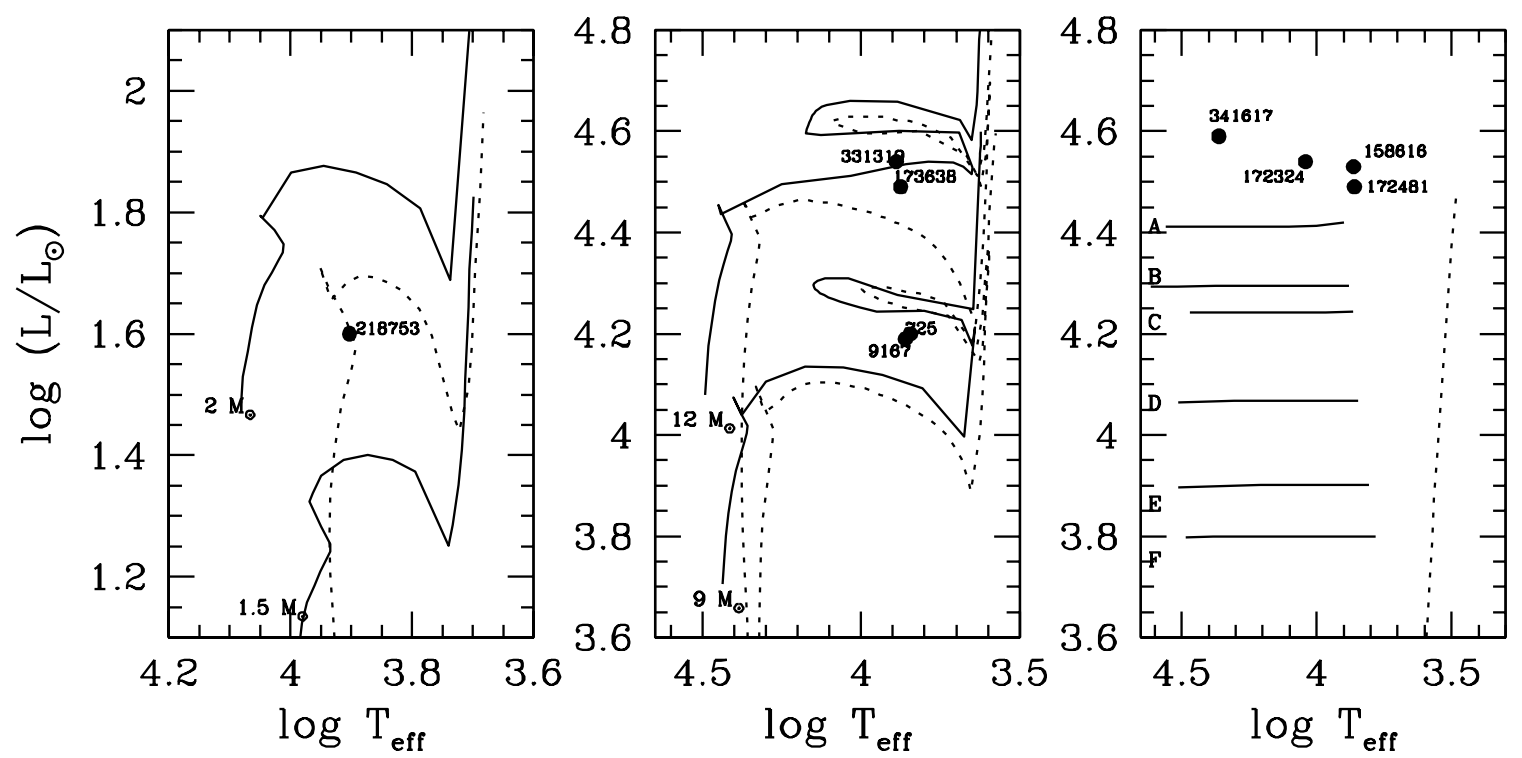

Fig. 5. H-R diagram showing the positions of sample stars along evolutionary tracks (continuous lines) and isochrones (dashed lines). $T_{\text {eff }}$ and $\log \left(L / L_{\odot}\right)$ are those in Table 2. The evolutionary tracks are from Schaller et al. (1992) and all isochrones from Bertelli et al. (1994). a) The star HD 218753 has $M \sim 1.5-2.0 M_{\odot}$ and age of $7.910^{8}$ yr. b) The two isochrones shown correspond to $3.1610^{7}$ and $2.010^{7} \mathrm{yr}$. See text for discussion. c) The post-AGB models are from Blöcker 1995 for ZAMS mass and core mass combinations $\left(M_{\text {zams }}, M_{\mathrm{H}}\right) ; \mathrm{A}:=\left(7 M_{\odot}, 0.940 M_{\odot}\right), \mathrm{B}:=\left(3 M_{\odot}, 0.836 M_{\odot}\right), \mathrm{C}:=\left(5 M_{\odot}, 0.836 M_{\odot}\right)$, $\mathrm{D}:=\left(4 M_{\odot}, 0.696 M_{\odot}\right), \mathrm{E}:=\left(3 M_{\odot}, 0.625 M_{\odot}\right), \mathrm{F}:=\left(3 M_{\odot}, 0.605 M_{\odot}\right)$. The isochrone shown corresponds to $2.510^{8} \mathrm{yr}$. See text for discussion

distances could be estimated without requiring the use of parallaxes. A straight comparison of distance moduli obtained from the Hipparcos parallaxes and from the P-L relation shows, in many cases, large differences. We have also noticed that Hipparcos parallaxes place some stars at unacceptable positions on the $\mathrm{H}-\mathrm{R}$ diagram given the derived atmospheric parameters and elemental abundances. For example, for stars HD 172324 and HD 172481 the parallax data suggest values of $\log \left(L / L_{\odot}\right)<2.7$. The situation is further complicated by unknown circumstellar reddening, which could be substantial for some of the stars, given their high infrared fluxes. Therefore we decided not to use individual parallaxes in the present context.

All pre-AGB evolutionary model tracks plotted in Fig. 5 are those of Schaller et al. (1992), while the postAGB models are from Blöcker (1995). All isochrones are from the work of Bertelli et al. (1994).

In terms of evolution, we could distinguish three groups among our sample stars. First, HD 158616, HD 172324, HD 172481, and HDE 341617 show clear indications of being post-AGB stars, these are shown in panel $c$ of Fig. 5. The post-AGB model sequences of Blöcker (1995) suggest that all four stars had initial ZAMS masses larger than $7 M_{\odot}$ and remnant or core mass of $\sim 1 M_{\odot}$. HD 158616 and HD 172481 might be starting their trajectory towards the white dwarfs region, i.e. they are near the zero age of central star evolution (Blöcker 1995), while HD 172324 is a bit more evolved. Since the evolution in this region of the $\mathrm{H}-\mathrm{R}$ diagram is very fast, they are all expected to become planetary nebulae within a few hundreds of years. HDE 341617 has been found to be in the early stages of PN (Arkhipova et al. 1999; Parthasarathy et al. 2000). Our remnant mass estimate of $\sim 1 M_{\odot}$ is substantially larger than $0.7 M_{\odot}$ estimated by Arkhipova et al. (1999) from the rate of temperature evolutionary change and a comparison with the theoretical rates from Blöcker's (1995) models. Our value is a consequence of the spectroscopic determination of $T_{\text {eff }}=23000 \mathrm{~K}$ and hence $\log \left(L / L_{\odot}\right)=4.6$ (Schmidt-Kaler 1982) as well as a comparison with the luminosities of Blöcker's (1995) models. While the Arkhipova et al. (1999) estimation is quite convincing the luminosity of the adopted model $\left(L / L_{\odot}\right)=4.0$ seems too low as it would imply $T_{\text {eff }} \sim 18000 \mathrm{~K}$ for bright giant star of luminosity class II. Such low a temperature is not supported by the detailed spectroscopic analyses. Thus an independent estimate of the luminosity seems necessary to settle the core mass of this star.

In the second evolutionary group we include the stars HD 725, HD 218753 and HD 331319. These are all moderately iron-deficient but otherwise show nearly solar abundances. The heliocentric radial velocities for HD 218753 and HD 331319 are small. They are most likely young massive disk supergiants or bright giants that have gone through some nuclear processing. This is suggested by the $\mathrm{C}$ depletion and $\mathrm{Na}$ enhancement, indicating the effect of CN processing and post first dredge-up stage. HD 725 is an interesting star since three Y II and one Ba II lines indicate mild enhancement of $s$-process elements. Since we count on very scarce number of lines of $s$-process elements, we can only say that the star shows signs of evolution beyond RGB. 
Table 14. Comparison of relevant $[\mathrm{X} / \mathrm{Fe}]$ ratios for program stars and well known post-AGB stars

\begin{tabular}{lccccccccccc}
\hline \multicolumn{1}{c}{ Star } & {$[\mathrm{Fe} / \mathrm{H}]$} & {$[\mathrm{C} / \mathrm{Fe}]$} & {$[\mathrm{O} / \mathrm{Fe}]$} & {$[\mathrm{Mg} / \mathrm{Fe}]$} & {$[\mathrm{Si} / \mathrm{Fe}]$} & {$[\mathrm{S} / \mathrm{Fe}]$} & {$[\mathrm{Ca} / \mathrm{Fe}]$} & {$[\mathrm{s} / \mathrm{Fe}]$} & $\mathrm{C} / \mathrm{O}$ & $\mathrm{reference}$ \\
\hline HD 725 & -0.29 & -0.08 & & +0.12 & +0.40 & +0.20 & +0.14 & +0.27 & & 1 \\
HD 9167 & -0.34 & & & -0.04 & & & +0.12 & +0.12 & \\
HD 172324 & -0.63 & -0.68 & +0.96 & -0.02 & +0.43 & +0.42 & & & +0.01 & 1 \\
HD 173638 & -0.08 & -0.09 & & +0.03 & +0.46 & & +0.09 & +0.14 & & 1 \\
HD 218753 & -0.19 & -0.30 & +0.05 & -0.09 & +0.23 & +0.36 & +0.11 & +0.14 & +0.21 & 1 \\
HD 331319 & -0.24 & -0.09 & +0.30 & -0.01 & +0.06 & +0.53 & -0.05 & -0.30 & +0.19 & 1 \\
\hline HD 158616 & -0.57 & +0.34 & +0.04 & +0.08 & +0.56 & +0.62 & +0.21 & +0.66 & +0.95 & 1 \\
HD 158616 & -0.70 & +0.64 & -0.04 & & +0.68 & +0.70 & +0.71 & +1.30 & +1.90 & 2 \\
HD 172481 & -0.61 & -0.01 & +0.04 & +0.51 & +0.54 & +0.58 & +0.34 & +0.52 & +0.43 & 1 \\
HR 7671 & -1.10 & -0.40 & -0.30 & +0.25 & +0.40 & +0.15 & +0.32 & +0.60 & +0.38 & 6 \\
\hline HD 187785 & -0.40 & +1.00 & +0.60 & +0.67 & +0.82 & +0.57 & +0.49 & +1.30 & +1.20 & 4 \\
HD 187785 & -0.60 & +1.00 & +0.70 & +0.38 & & +0.26 & +0.49 & +1.10 & \\
HD 56126 & -1.00 & +1.08 & +0.63 & +0.97 & +0.95 & +0.63 & +0.46 & +1.78 & +1.35 & 3 \\
HD 56126 & -1.00 & +1.10 & +0.80 & +0.06 & & +0.40 & -0.11 & +1.50 & 11 \\
IRAS 04296+3429 & -0.60 & +0.80 & & & +0.79 & +0.43 & +0.18 & +1.50 & 11 \\
IRAS 05341+0852 & -0.80 & +1.00 & +0.60 & & +0.59 & +0.28 & +0.08 & +2.20 & 1 \\
IRAS 22223+4237 & -0.30 & +0.30 & -0.10 & & +0.29 & +0.04 & -0.17 & +0.90 & 11 \\
IRAS 23304+6147 & -0.80 & +0.90 & +0.20 & & +0.79 & +0.56 & +0.29 & +1.60 & 11 \\
\hline HR 4049 & $<-3.2$ & $>+3.0$ & $>+2.7$ & $>+1.7$ & $>+0.40$ & $>+3.0$ & $<-2.1$ & & +0.95 & 5 \\
HD 44179 & -3.30 & +3.30 & +2.90 & +1.2 & +1.50 & +3.00 & & +0.20 & +1.20 & 2 \\
HD 46703 & -1.57 & +0.98 & +1.10 & +0.09 & -0.38 & +1.20 & +0.02 & -0.49 & +0.74 & 7,8 \\
HD 52961 & -4.80 & +4.40 & +4.20 & & & +3.80 & & +0.60 & +0.76 & 2 \\
HD 70379 & -0.31 & +0.42 & +0.38 & +0.01 & +0.47 & +0.34 & -0.17 & +0.20 & +0.47 & 9 \\
BD+39 4926 & -2.85 & +2.45 & +2.75 & +1.35 & +1.15 & +2.95 & -0.75 & +1.60 & +0.25 & 10 \\
\hline
\end{tabular}

References: 1 - This work, 2 - Van Winckel (1995) , 3 -Klochkova (1995), 4 - Van Winckel et al. (1996), 5 - Lambert et al. (1988), 6 - Luck et al. (1990), 7 - Luck \& Bond (1984), 8 - Bond \& Luck (1987), 9 - Reddy (1996), 10 - Kodaira (1973), 11 - Van Winckel \& Reyniers (2000).

The tracks and isochrones in Figs. 5a and 5b suggest $M \sim 1.5-2.0 M_{\odot}$ and age of $7.910^{8}$ yr for HD 218753 and $M \sim 11 M_{\odot}$ and age of $2.210^{7} \mathrm{yr}$ for HD 331319. From tracks in Fig. 5b we estimate a mass of $M \leq 9 M_{\odot}$ and age of $2.510^{7}$ yr. Its rather large heliocentric radial velocity of $-57 \mathrm{~km} \mathrm{~s}^{-1}$ calls for attention, however, a calculation of the galactocentric motion indicates that the star moves on the galactic plane and has a mildly eccentrical galactic orbit. Its radial velocity could owe its origin to pulsations and/or orbital motions, although variability has not been reported.

The last two stars in our sample, HD 9167 and HD 173638, display, with few exceptions, solar abundances and no signs of nuclear processing. They are probably evolving very near the giant branch. The estimated masses and ages from Fig. 5b are respectively $M \sim 10 M_{\odot}$ and $2.610^{7} \mathrm{yr}$ and $M \sim 12 M_{\odot}$ and $1.710^{7} \mathrm{yr}$. The only peculiarity of HD 9167 is its high radial velocity of $-45.7 \mathrm{~km} \mathrm{~s}^{-1}$ however, like HD 725, its galactocentric orbit seems to be on the galactic plane and mildly eccentric. Also the possibility, that their observed radial velocities are attained from pulsation and/or orbital motion cannot be discarded.

It should be noted that although HD 172481 and HD 158616 are post-AGB stars, they do not show the effect of selective removal of condensable elements such as $\mathrm{Fe}$ and Sc, observed in some well-known post-AGB stars like HR 4049, and HD 52961 and RV Tau stars of subclass B (Giridhar et al. 2000 and references therein). While studying a sample of RV Tau stars, these authors had noticed a strong dependence on temperature for the selective removal of refractory elements to occur. The effect is very prominent at temperature range 5500 to $6000 \mathrm{~K}$ and declines for lower temperatures. For stars cooler than $5000 \mathrm{~K}$ the effect was barely perceptible. At temperatures higher than $7000 \mathrm{~K}$, we expect the effect to be larger. It is indeed true for HR 4049 (Lambert et al. 1988) which has $T_{\text {eff }}=7500 \mathrm{~K}$, i.e. similar to HD 158616 and HD 172481 . However, for these two stars we did not see any indication of dust condensation and subsequent removal of grainforming elements. HD 158616 is a carbon-rich post-AGB star similar to HD 56126 (Klochkova 1995) and HD 187785 (Van Winckel et al. 1996) also showing significant enhancement of $s$-process elements.

Stars like HR 4049, HD 52961 and RV Tau stars of subclass B show $\mathrm{C} / \mathrm{O} \leq 1$ and mild $s$-process enhancement. As a matter of fact, most stars showing abundance peculiarities caused by dust condensation have $\mathrm{C} / \mathrm{O} \leq 1$. Stars HR 4049, HD 44179, HD 46703, HD 52961 and $\mathrm{BD}+39^{\circ} 4926$ possibly belong to this subgroup. For these objects, since Fe gets locked in grains, $[\mathrm{S} / \mathrm{H}]$ is considered a better indicator of metallicity. For these stars $[\mathrm{S} / \mathrm{H}]$ ranges 
between +0.1 to -1.0 dex with a mean around -0.4 dex. In other words, they are mildly metal-deficient. Carbonrich post-AGB stars with enhanced $s$-process elements, like HD 158616, HD 56126 and HD 187785 have [Fe/H] (which would be a true reflection of their metallicity since these stars are not affected by dust condensation) in the range -0.4 to -1.0 dex. These values are not radically different from those found for the subgroup having dustgrain condensation and $\mathrm{C} / \mathrm{O} \leq 1$. We, therefore, do not visualize large differences in their ages though the O-rich phase in the AGB is expected to precede the C-rich phase.

The abundances of hot post-AGB stars studied by Conlon et al. (1993a) and McCausland et al. (1992) bear close resemblance to HD 172324. The hot post-AGB stars show strong deficiency of carbon and significant oxygen enrichment. These stars probably belong to a subgroup of post-AGB star that have evolved without experiencing third dredge-up. This carbon deficiency is also found in the proto-Planetary Nebula HDE 341617 (see Table 13). Caution is however needed with C II spectra since they are known to show large non-LTE effects (Eber \& Butler 1988; Takeda \& Takada-Hidai 1994). McCausland et al. (1992) have discussed at length two scenarios to explain the carbon deficiency. HBB occuring during interpulse phase could cause the production of ${ }^{14} \mathrm{~N}$ at the expense of ${ }^{12} \mathrm{C}$. However, overabundance of He like the one found in the SMC planetary nebula SMP 28 is not evident for HD 172324 and HD 341617 to make HBB the sole mechanism responsible for carbon deficiency. Another possibility suggested by McCausland et al. (1992) that the carbon deficiency might be inherent to the precursor itself is quite attractive. To substantiate their argument they pointed out the carbon-poor stars HR 4912 and HR 7671 as possible precursors to more evolved carbon-poor hot postAGB stars. HR 4912 was included in our recent work and we found $[\mathrm{C} / \mathrm{H}]=-1.27$ (Giridhar et al. 1997) in good agreement with $[\mathrm{C} / \mathrm{H}]$ of -1.15 found by Lambert et al. (1983). HR 7671 has $[\mathrm{C} / \mathrm{H}]$ of -1.53 (Luck et al. 1990). It seems therefore that HD 172324 and HDE 341617 might form a special carbon-poor post-AGB stars evolutionary sequence. Search for carbon-poor objects in all temperature ranges may help in finding the precursors or successors of these objects.

\section{Conclusions}

We have found a new post-AGB star HD 172481 for which abundance analysis had not been carried out before, however the referee pointed out in a rather late stage of revision, the then unpublished work by Reyniers \& Van Winckel (2001) where an independent abundance analysis has been carried out. We have highlighted a comparison of results in Sect. 5.5 and found both analyses to be in fairly good agreement. We have done a more complete analysis of HD 158616. This star can be now considered a postAGB star beyond any doubt. Among the post-AGB stars, the number of stars showing $\mathrm{C} / \mathrm{O} \sim 1$ or greater and also enhancement of $s$-process elements are very few. There are clear indications of stars having experienced the third dredge-up. The stars HD 158616 and HD 172481 belong to this important class. C/O of HD 172481 might have been prevented from exceeding one by HBB. The same may be responsible for large $\mathrm{Li}$ abundance but other possibilities like binarity cannot be ruled out. A long-term light and radial velocity monitoring of this object is planned for the future.

We found a very likely hot post-AGB candidate in HD 172324 but will feel more confident of its status after important elements like $N$ are included and a more comprehensive study is made. Continuous monitoring of its spectrum in the $\mathrm{H}_{\alpha}$ region is required to detect activity possibly related to stellar pulsations.

HD 218753 and HD 331319 have passed the giant branch and are in the He-core and H-shell burning stages. HD 9167 and HD 173638 essentially show solar abundance. HD 725 and HD 9167 show large radial velocities, however their galactocentric orbits are on the galactic plane and mildly eccentric, thus they are most likely massive and young disk stars. However, their large radial velocity could also be due to pulsations and/or orbital motions.

In the proto-Planetary Nebula HDE 341627 the He lines show two velocity components possibly indicating velocity stratification. The emission lines appear to have weakened since 1993.

Acknowledgements. AAF acknowledges Commission 38 of the IAU for a travel grant and the Indian Institute of Astrophysics for hospitality and financial support. We thank David Yong for getting us two spectra of HD 172481. We are also indebted to Ms. T. Sivarani for her help in identifying the lines for HDE 341617. We are grateful to the referee, Dr. R. Gallino, for helpful discussions and suggestions. This project has been supported at different stages by grants from DGAPA-UNAM (IN113599) and CONACyT (Mexico) (E130.2060) and CNRS (France).

\section{References}

Arellano Ferro, A. 1985, RMA\&A, 11, 113

Arellano Ferro, A., Giridhar, S., \& Goswami, A. 1991, MNRAS, 250,1

Arellano Ferro, A., \& Mantegazza, L. 1996, A\&A, 315, 542

Arkhipova, V. P., Ikonnikova, N. P., Noskova, R. I., et al. 1999, Astron. Lett., 25, 25

Baranne, A., Queloz, D., Mayor, M., et al. 1996, A\&AS, 119, 373

Bertelli, G., Bressan, A., Chiosi, C., Fagotto, F., \& Nasi, E. 1994, A\&AS, 106, 275

Bidelman, W. P. 1990, in Luminous High-Latitude Stars, ASP Conf. Ser., 45, 47

Blöcker, T. 1995, A\&A, 299, 755

Bogart, E. 1994, Ph.D. Thesis, Katholieke Uni. Leuven

Bond, H. E., \& Luck, R. E. 1987, ApJ, 312, 203

Boothroyd, A. I., Sackmann, I. J., \& Ahern, S. C. 1993, ApJ, 416,762

Boothroyd, A. I., \& Sackmann, I. J. 1999, ApJ, 510, 232

Boyarchuck, A. A., Lyubimkov, L. S., \& Sakhibullin, N. A. 1985, Astrophys., 22, 203 
Bravo Alfaro, H., Arellano Ferro, A., \& Schuster, W. J. 1997, PASP, 109, 958

Busso, M., Gallino, R., \& Wasserburg, G. J. 1999, ARA\&A, 37, 239

Busso, M., Lambert, D. L., Beglio, L., \& Gallino, R. 1995, ApJ, 446, 775

Conlon, E. S., Dufton, P. L., McCausland, R. J. H., \& Keenan, F. P. 1993a, ApJ, 408, 593

Conlon, E. S., McCausland, R. J. H., Dufton, P. L., \& Keenan, F. P. 1993b, in Luminous High-Latitude Stars, ASP Conf. Ser., 45, 146

Cowley, A., Cowley, Ch., Jaschek, M., \& Jaschek, C. 1969, AJ, 74,375

Cramer, N., \& Maeder, A. 1979, A\&A, 78, 305

Crawford, D. 1970, AJ, 75, 624

Crawford, D. 1979, AJ, 84, 1858

Decin, L., Van Winckel, H., Waelkens, C., \& Bakker, E. J. 1998, A\&A, 332, 928

Didelon, P. 1982, A\&AS, 50, 199

Downes, R. A., \& Keyes, C. D. 1988, AJ, 96, 777

Eber, F., \& Butler, K. 1988, A\&A, 202, 153

Edvardsson, B., Andersen, J., Gustafsson, B., et al. 1993, A\&A, 275,101

Feast, M. W., \& Catchpole, R. M. 1997, MNRAS, 286, L1

Gallino, R., Arlandini, C., Busso, M., et al. 1998, ApJ, 497, 388

Gigas, D. 1986, A\&A, 165, 170

Giridhar, S., \& Arellano Ferro, A. 1989, JA\&A, 10, 47

Giridhar, S., \& Arellano Ferro, A. 1995, RMA\&A, 31, 23

Giridhar, S., Arellano Ferro, A., \& Parrao, L. 1997, PASP, 109, 1077

Giridhar, S., Lambert, D. L., \& Gonzalez, G. 2000, ApJ, 531, 521

Gonzalez, G., Lambert, D. L., \& Giridhar, S. 1997, ApJ, 479, 427

Grevesse, N., Noels, A., \& Sauval, A. J. 1996, ASP Conf. Ser., 99, 117

Griffin, R. F., \& Redman, R. O. 1960, MNRAS, 120, 287

Hauck, B., \& Mermilliod, M. 1998, A\&AS, 129, 431

Klochkova, V. G. 1995, MNRAS, 272, 710

Kodaira, K. 1973, A\&A, 22, 273

Kraft, P. R., Sneden, C., Langer, G. E., \& Prosser, C. F. 1992, AJ, 104, 645

Kurucz, R. L. 1993, ATLAS9 Stellar atmosphere Programs and $2 \mathrm{~km} \mathrm{~s}^{-1}$ grid CDRoM, vol. 13 (Cambridge: Smithsonian Astrophysical Observatory)

Lambert, D. L. 1992, in Instabilities in Evolved Super and Hypergiants, ed. C. de Jager, \& H. Nieuwenhuijzen (Amsterdam: North-Holland), 156

Lambert, D. L., Heath, J. E., Lemke, M., \& Drake, J. 1996, ApJS, 103, 183

Lambert, D. L., Hinkle, K. H., \& Luck, R. E. 1988, ApJ, 333, 917

Lambert, D. L., Luck, R. E., \& Bond, H. E. 1983, PASP, 95, 413

Langer, G. E., Hoffman, R., \& Sneden, C. 1993, PASP, 105, 301

Lattanzio, J. C. 1997, in 2nd Oak Ridge Symp. on Atomic and Nuclear Astrophysics (IOP Publishing Ltd.)

Luck, R. E., \& Bond, H. E. 1983, ApJL, 271, L75

Luck, R. E., \& Bond, H. E. 1984, ApJ, 279, 729

Luck, R. E., \& Bond, H. E. 1985, ApJ, 292, 559
Luck, R. E., Bond, H. E., \& Lambert, D. L. 1990, ApJ, 357, 188

McErlean, N. D., Lennon, D. J., \& Dufton, P. L. 1999, A\&A, 349,553

McCarthy, J. K., Sandiford, B. A., Boyd, D., \& Booth, J. 1993, PASP, 105, 881

McCausland, R. J. H., Conlon, E. S., Dufton, P. L., \& Keeenan, F. P. 1992, ApJ, 394, 298

Morgan, W. W., \& Roman, N. G. 1950, ApJ, 112, 362

Mowlavi, N. 1999, New Astr. Rev., 43, 389

Napiwotzki, R., Shönberner, D., \& Wenske, V. 1993, A\&A, 268,653

Olsen, E. H. 1983, A\&AS, 54, 55

Olnon, F. M., Baud, B., Habing, H. J., et al. 1984, ApJL, 278, L41

Parthasarathy, M, García-Lario, P., Sivarani, T., Manchado, A., Sanz Fernandez de Córdoba, L. 2000, A\&A, 357, 241

Perry, C. L. 1969 AJ, 74, 705

Reddy, B. E. 1996, Ph.D. Thesis, Bangalore University, India

Reyniers, M., \& Van Winckel, H. 2001, A\&A, 365, 465

Sackmann, I. J., \& Boothroyd, A. I. 1992, ApJ, 392, L71

Schaller, G., Schaerer, D., Meynet, G., \& Maeder, A. 1992, A\&AS, 96, 269

Schmidt-Kaler, Th. 1982, in Numerical Data and Functional Relationships in Science and Technology, ed. K. Schaifers, \& H. H. Vogt (Springer-Verlag)

Schwarzschild, M., \& Härm, R. 1965, ApJ, 142, 885

Smith, V. V., Plez, B., \& Lambert, D. L. 1995, ApJ, 441, 735

Sneden, C. 1973, Ph.D. Thesis, Univ. of Texas at Austin

Sneden, C., Kraft, R. P., Prosser, C. F., \& Langer, G. E. 1991, AJ, 102, 2001

Stephenson, C. B. 1986, ApJ, 300, 779

Straniero, O., Chieffi, A., Limongi, M., et al. 1997, ApJ, 478, 332

Straniero, O., Gallino, R., Busso, M., Chieffi, A., \& Raiteri, C. M. 1995, ApJ, 440, L85

Takeda, Y., \& Takada-Hidai, M. 1994, PASJ, 46, 395

Takeda, Y., Takada-Hidai, M., \& Kotake, J. 1996, PASJ, 48, 753

Takeda, Y., \& Takada-Hidai, M. 1998, PASJ, 50, 629

Thévenin, F. 1989, A\&AS, 77, 137

Thévenin, F. 1990, A\&AS, 82, 179

Travaglio, C., Galli, D., Gallino, R., et al. 1999, ApJ, 521, 691

Tull, R. G., MacQueen, P. J., Sneden, C., \& Lambert, D. L. 1995, PASP, 107, 251

van der Veen, W., \& Habing, H. J. 1988, A\&A, 194, 125

Van Winckel, H. 1995, Ph.D. Thesis, Katholieke Univ. Leuven, The Netherlands

Van Winckel, H. 1997, A\&A, 319, 561

Van Winckel, H., \& Reyniers, M. 2000, A\&A, 354, 135

Van Winckel, H., Waelkens, Ch., \& Waters, L. B. F. M. 1996, A\&A, 306, L37

Venn, K. A. 1995a, ApJS, 99, 659

Venn, K. A. 1995b, ApJ, 449, 832

Volk, K. M., \& Kwok, S. 1989, ApJ, 342, 345

Wallerstein, G., Iben, I. Jr., Parker, P., et al. 1997, Rev. Mod. Phys., 69, 995

Weigert, A. 1966, Z. Astrophys., 64, 395

Wiese, W. L., Führ, J. R., \& Deters, T. M. 1996, J. Phys. Chem. Ref. data Monogr., No. 7

Wheeler, J. C., Sneden, Ch., \& Truran, J. W. 1989, ARA\&A, 27,279 